\title{
Transcriptome profiling of the cold response and signaling pathways in Lilium lancifolium
}

\author{
Jingmao Wang1, Yang Yang², Xiaohua Liu', Jie Huang ${ }^{1}$, Qing Wang ${ }^{1}$, Jiahui Gu and Yingmin Lu*
}

\begin{abstract}
Background: Lilium lancifolium, a very important cold-resistant wild flower for lily cold resistance breeding, is widely distributed in southwestern and northeastern China. To gain a better understanding of the cold signaling pathway and the molecular metabolic reactions involved in the cold response, we performed a genome-wide transcriptional analysis using RNA-Seq.

Results: Approximately 104,703 million clean 90- bp paired-end reads were obtained from three libraries (CK 0 h, Cold-treated $2 \mathrm{~h}$ and $16 \mathrm{~h}$ at $\left.4^{\circ} \mathrm{C}\right) ; 18,736$ unigenes showed similarity to known proteins in the Swiss-Prot protein database, and 15,898, 13,705 and 1849 unigenes aligned to existing sequences in the KEGG and COG databases (comprising 25 COG categories) and formed 12 SOM clusters, respectively. Based on qRT-PCR results, we studied three signal regulation pathways — the $\mathrm{Ca}^{2+}$ and $\mathrm{ABA}$ independent/dependent pathways — that conduct cold signals to signal transduction genes such as LIICE and LICDPK and transcription factor genes such as LIDREB1/CBF, LIAP2/EREBP, LINAC1, LIR2R3-MYB and LIBZIP, which were expressed highly in bulb. LIFAD3, LIB-amylase, LIP5CS and LICLS responded to cold and enhanced adaptation processes that involve changes in the expression of transcripts related to cellular osmoprotectants and carbohydrate metabolism during cold stress.

Conclusions: Our study of differentially expressed genes involved in cold-related metabolic pathways and transcription factors facilitated the discovery of cold-resistance genes and the cold signal transcriptional networks, and identified potential key components in the regulation of the cold response in $L$ lancifolium, which will be most beneficial for further research and in-depth exploration of cold-resistance breeding candidate genes in lily.
\end{abstract}

Keywords: Lilium lancifolium, RNA-Seq, Transcriptome, Cold response, Signaling pathway, Biochemical mechanism

\section{Background}

Plants have a remarkable ability to cope with highly variable environmental abiotic stresses, including cold, drought, heat, salinity and nutrient deficiencies. Nevertheless, these stresses together represent the primary cause of plant injuries and losses worldwide, reducing the ornamental value and production of most major plants and crops by more than $50 \%$ [1]. As a wild coldresistant plant, Lilium lancifolium is mainly distributed in the North Temperate Zone, where the winter temperate can fall as low as $-35^{\circ} \mathrm{C}$, but it can survive exposure, acclimate to low or freezing temperatures and continuously germinate in the next spring. In addition, studies

\footnotetext{
* Correspondence: luyingmin@bjfu.edu.cn

${ }^{1}$ College of Landscape Architecture \& China National Engineering Research Center for Floriculture, Beijing Forestry University, No.35 Qinghua East Road Haidian District, Beijing 100083, China

Full list of author information is available at the end of the article
}

have shown its capacity for resisting heat, drought and changing soil salinity. Nevertheless, the decline of L. lancifolium is gradually becoming more serious in recent years with the deterioration of its ecological environment. Therefore, further protection and a better understanding of the gene expression profile of L. lancifolium under cold stress is imperative, and it could be an ideal model to study cold tolerance mechanisms and signaling regulation for improving the quality of cold resistance in other plants using molecular biological techniques.

Cold responses have been observed in many plants, which initiate reactions of the freezing tolerance of plants after low temperature stress, including Arabidopsis [2], Oryza sativa [3], Triticum aestivum [4], and Ammopiptanthus mongolicus [5]. The initiation of most stress treatments correlates with a cytosolic calcium release, in some cases with stimulus-specific patterns of oscillation [6]. In addition, stimulus-specific changes in gene expression are 
often observed alongside a set of shared stress responses. For example, in a survey of 1, 300 Arabidopsis genes, the majority of cold and drought stress regulated genes were observed in the shared stress response [7]. Together, these observations support the hypothesis that a common set of signal transduction pathways are triggered during many stress responses. Many of the biochemical, molecular and physiological changes that occur during the cold response are considered to be important in the induction of freezing tolerance. During this process, plants alter the expression of certain genes as well as the biosynthesis of amino acids and soluble sugars [8]. At the gene expression level, DNA microarray analysis studies have revealed that exposure of Arabidopsis plants to a low temperature of $4^{\circ} \mathrm{C}$ resulted in up- or down-regulation of hundreds to thousands of cold-regulated $(C O R)$ genes, and that many of the cold inducible genes are linked with the accumulation of osmolytes, cryoprotectants, antioxidant detoxification enzymes, chaperones, transporters, dehydrins, late embryogenesis abundant $(L E A)$ proteins and enzymes involved in lipid, carbohydrate and secondary metabolites, and in abscisic acid (ABA) and jasmonic acid (JA) biosynthesis [9].

Traditional cloning and genetic transformation methods are expensive and time consuming. In recent years, the development of novel high-throughput sequencing technologies, such as Solexa/Illumina RNA-Seq (RNA sequencing) and digital gene expression (DGE) has provided an opportunity to explore cold resistance and signaling-associated genes in different species by de novo assembly or mapping, and also facilitated rapid identification and analysis of the vast majority of transcriptomes [10]. Transcriptome sequencing is an efficient means to generate functional genomic data for non-model organisms or those with genome characteristics prohibitive to whole-genome sequencing [11]. Illumina/Solexa has been successfully applied to the transcriptome sequencing of many plant species, including Populus euphratica [12], Aegilops variabilis [13], Aechmea fasciata [14], Brassica napus [15], Zea mays [16], Arachis hypogaea [17], and Picrorhiza kurrooa [18]. For instance, transcriptome profiling of grapefruit flavedo following exposure to low temperature and conditioning treatments has increased our understanding of the principal molecular components involved in chilling tolerance and susceptibility [19]. Using Illumina/Solexa, we found that the study of differentially expressed genes involved in coldrelated metabolic pathways and transcription factors could facilitate the discovery of cold-resistance genes for the desert shrub Ammopiptanthus mongolicus [20]. The transcriptomic analysis of Aechmea fasciata treated with ethylene has been revealed part of the ethylene signaling pathway and flowering process [14]. Genome-wide analysis approaches have been used to elucidate gene expression in response to drought stress in Populus simoniia [21].
However, few studies have been carried out to date on the cold-related metabolic pathways and transcription factors in L. lancifolium. Such studies would bridge the physiological and anatomical changes during cold acclimation with molecular data. Here, we present three cDNA libraries (two cold-treated and a control) of living L. lancifolium leaves which were subjected to short-term cold $\left(4^{\circ} \mathrm{C}\right)$ stress treatment and describe the short-term cold response ( 0 to $16 \mathrm{~h}$ ) of $L$. lancifolium using the next-generation Illumina/Solexa sequencing technology, and also compared the long-term cold acclimation ( 1 to 20d) of L. lancifolium and Oriental hybrids using anatomical and physiological analyses and biochemistry experiments. We had two specific objectives. First, to identify genes that change expressions in a stressspecific fashion and reveal the transcription factors that change in the key transcriptional, finding development networks and signal pathways in the response to cold stress. Second, to identify the expression of various genes that are co-regulated during the biological processes, such as intercellular osmoprotectant biosynthesis and biodegradation of carbohydrates of shared stress responses. This global view illustrates the "fluid" nature of the transcriptome and the challenge we face in understanding the complexity of any given stress response. In particular, the analyses on differentially expressed genes under cold stress furthers our understanding of the cold response mechanism of L. lancifolium, and these coldrelated genes should also contribute to providing a method of developing cold-tolerant plants through genetic manipulation.

\section{Results}

\section{High-throughput transcriptome sequencing and read assembly}

To identify the number of genes involved in the transcriptome, a cDNA sample was prepared from an equal mixture of total RNA isolated from leaves for three libraries (cold-treated $2 \mathrm{~h}, 16 \mathrm{~h}$ and control $0 \mathrm{~h}$ samples), which were sequenced using the Illumina HiSeq 2000. We obtained approximately 74 million raw reads for the two cold-treated samples (CT2h and CT16h) and 41 million for the control sample (CKOh). We discarded low-quality reads, which contained adapters and unknown or low-quality bases, according to our bioinformatics analysis. After stringent quality checks and data cleaning, we obtained 115,421,520 raw reads containing a total of $11.6 \mathrm{~Gb}$ nucleotides. The average read size, Q20 percentage (sequencing error rate $<1 \%$ ), and GC (guanine + cytosine) percentage were $90 \mathrm{bp}, 98.1 \%$, and $43.7 \%$, respectively. Based on the high quality reads, 46,516 contigs were assembled with an average length of $793 \mathrm{bp}$. With paired-end joining and gap-filling, the contigs were further assembled into 39,154 scaffolds with an 
average length of $951 \mathrm{bp}$, including 11478 scaffolds larger than $1000 \mathrm{bp}$. After local assembly with the unmapped ends to fill in the small gaps within the scaffolds, the de novo assembly yielded 37,843 unigenes with an average length of $971 \mathrm{bp}$ (Tables 1 and 2). To demonstrate the quality of sequencing data, ten unigenes were randomly selected and ten pairs of primers were designed for qRT-PCR, and then the products were confirmed by biological Sanger sequencing.

To validate and annotate the of assembled unigenes, using E-value < 1e-5, they were blast searched against the UniProt (date: 2013.04) and Swiss-Prot protein database (date: 2013.05) (http://www.expasy.ch/sprot) which has the largest and most detailed protein annotation database, including 24,889,084 proteins. The results indicated that among the 37,843 unigenes, 18,736 (49.5\% of the total) had significant similarity to known proteins in the Swiss-Prot database. The lack of L. lancifolium genome and EST information meant that 19.107 (50.5\% of the total) unigenes had no Swiss-Prot annotation (Table 2).

\section{Gene annotation and functional classification}

To further evaluate the completeness of our transcriptome library and the effectiveness of our annotation process, we randomly searched the annotated sequences for genes with COG (Clusters of orthologous groups) classifications. Of 18,736 Swiss-Prot hits, 13,705 sequences had a COG classification. Among the $25 \mathrm{COG}$ categories, the cluster for 'Signal transduction mechanisms' (8332, 17.54\%) represented the largest group, followed by 'Cytoskeleton' (7078, 14.89\%) and 'General function prediction only' (4239, 8.92\%). The 'defense mechanisms' (190, 0.39\%) and 'extracellular structures' (147, 0.30\%) categories, represented the smallest groups (Figure 1). Compared with the control sample, the mostabundant cluster in the cold treated samples was 'Signal transduction mechanisms', indicating that STM genes play a vital regulation role in the L. lancifolium cold senescence and stress responses.

The Gene ontology (GO) assignments system was also used to classify the functions of the predicted L. lancifolium genes. Based on sequence homology, the 18,736 sequences were categorized into 53 functional groups (Figure 2). The unigenes were then assigned into three main categories: biological process, cellular component, and molecular function, with 7901 (42.17\%), 2338 (12.47\%), and 4459 (23.79\%) unigenes, respectively, assigned to each. In each of the three main GO classifications, the 'metabolic process', 'cellular process', and 'catalytic activity' terms, were dominant. We also noticed a high percentage of genes from the 'cellular process', 'cell part', and 'binding' categories, but few from 'cell junction', 'symplast', 'extracellular region part' and 'locomotion' (Figure 2). The GO analysis indicated that a great number of identified genes were associated with various biological processes and molecular functions under low temperature. About 5331 sequences were annotated as belonging to the 'metabolic process' category, which may allow for the identification of novel genes involved in secondary metabolism pathways in cold acclimation. The 'cellular component part' suggested that the membrane and cell junction could function in the later cold resistance process (Figure 2).

The KEGG (Kyoto Encyclopedia of Genes and Genomes) is a database resource for understanding the high-level functions and utilities of biological systems, such as the cell, the organism and the ecosystem, from molecular-level information, especially large-scale molecular datasets generated by genome sequencing and other high-throughput experimental technologies (see the release notes for new and updated features). The pathway database records networks of molecular interactions in cells, and variants specific to particular organisms. Based on a comparison against the KEGG database using BLASTx with an E-value cutoff of $<10^{-5}$, of the 37,843 unigenes, 15,898 (42\%) had significant matches in the database and were assigned to 119 KEGG pathways (Table 3). The most represented pathways were 'metabolism pathways' (1784 members, 11.23\%), 'biosynthesis of secondary metabolites' (946 members, 5.95\%), and 'Microbial metabolism in diverse environments' (400 members, 2.51\%) (Table 3). These annotations provide a valuable resource for investigating the processes, functions, and pathways involved in the short-term cold response and long-term cold acclimation.

\section{Changes in gene expression profiles among the different cold stress stages}

To investigate the annotated transcriptome assembly and gain statistical confirmation of the differences in gene expression that served as a reference for RNA-Seq

Table 1 Overview of the sequencing and assembly

\begin{tabular}{|c|c|c|c|c|c|c|c|c|}
\hline Sample ID & Raw reads (MB) & Raw bases (GB) & Q20 value (\%) & $\begin{array}{l}\text { Raw } \\
\text { reads }\end{array}$ & $\begin{array}{l}\text { Quality } \\
\text { trimed }\end{array}$ & $\begin{array}{l}\text { Adaptor } \\
\text { trimed }\end{array}$ & $\begin{array}{c}\text { Number Clean } \\
\text { reads }\end{array}$ & $\begin{array}{c}\text { Clean } \\
\text { ratio }\end{array}$ \\
\hline Control-0 h & 36.5 & 3.7 & $98 . \%$ & $36,521,060$ & $34,375,258$ & $33,947,498$ & $33,259,676$ & $91.1 \%$ \\
\hline Treatment-2 h & 37.1 & 3.7 & $98.1 \%$ & $37,063,886$ & $35,088,672$ & $34,672,716$ & $33,979,556$ & $91.7 \%$ \\
\hline Treatment-16 h & 41.8 & 4.2 & $98.1 \%$ & $41,836,574$ & $38,733,184$ & $38,229,688$ & $37,465,870$ & $89.6 \%$ \\
\hline
\end{tabular}


Table 2 Summary for the Lilium lancifolium transcriptome

\begin{tabular}{lccccccccccc}
\hline Statistics & Counts & $\begin{array}{c}\text { Total Length } \\
\mathbf{( b p )}\end{array}$ & $\begin{array}{c}\mathbf{N} 25 \\
\mathbf{( b p )}\end{array}$ & $\begin{array}{c}\text { N50 } \\
(\mathbf{b p})\end{array}$ & $\begin{array}{c}\mathbf{N 7 5} \\
\mathbf{( b p )}\end{array}$ & $\begin{array}{c}\text { Average } \\
\text { length }\end{array}$ & $\begin{array}{c}\text { Longest } \\
\text { (bp) }\end{array}$ & $\begin{array}{c}\mathbf{N} \\
\%\end{array}$ & $\begin{array}{c}\text { GC } \\
\%\end{array}$ & $\begin{array}{c}\text { Annotation } \\
\text { counts }\end{array}$ & $\begin{array}{c}\text { Annotation } \\
\text { ratio \% }\end{array}$ \\
\hline Contigs & 46,516 & $36,896,235$ & 1,706 & 962 & 582 & 793 & 14,570 & 0.9 & 43.7 & & \\
Primary uniGene & 39,154 & $37,242,700$ & 1,919 & 1,106 & 648 & 951 & 14,570 & 0.9 & 43.7 & & \\
Final uniGene & 37,843 & $36,756,285$ & 1,963 & 1,146 & 663 & 971 & 14,570 & 1.0 & 43.6 & 18,736 & 49.5 \\
\hline
\end{tabular}

profiling of stage-specific expression, we conducted a small RNA-Seq experiment using tangential cryosections of CKOh, CT2h and CT16h of L. lancifolium and mapped the resulting reads to our reference transcriptome. To minimize false positives and negatives, we concluded that a statistical analysis was reliable when applied to genes with an RPKM value $\geq 2$ in at least one of the three stages. It should be noted that this statistical significance was based on expected sampling distributions. To determine which of the 37,843 genes were differentially expressed among the three stages, we filtered with an FDR $\leq 0.001$ and $\mid \log _{2}$ (ratio) $\mid \geq 2$; the expression of 2755 DEGs was found to be significantly changed during the three stages. On the one hand, some gene were down-regulated from the $0 \mathrm{~h}$ to $2 \mathrm{~h}$, but upregulated obviously at $16 \mathrm{~h}$. On the other hand, some genes showed increased quantitative expression at $2 \mathrm{~h}$, but decreased transcript abundance at the $16 \mathrm{~h}$ stage. To identify genes showing a significant change in expression during different cold stress stages, the differentially expressed tags between the three samples were identified using an algorithm developed from the heat-map. Some genes were immediately expressed at the initial stage of cold stress, while others were up-regulated subsequently indicating that transcription factors induced the expression of cold-related genes during the regulation of cold signaling (Figure 3A).

In addition, we compared the $\mathrm{Ck} 0 \mathrm{~h}$ and $\mathrm{CT} 2 \mathrm{~h}$ libraries, and 343 variable genes were found a total of 115 upregulated and 228 down-regulated genes were detected between the two L. lancifolium libraries. There were also 326 up-regulated and 828 down-regulated genes between the COh and T16h libraries, 410 up-regulated and 848 downregulated genes between the $\mathrm{C} 2 \mathrm{~h}$ and $\mathrm{T} 16 \mathrm{~h}$ libraries (Figure 3B). This suggests that the differentiation of expressed genes between $\mathrm{C} 2 \mathrm{~h}$ and $\mathrm{T} 16 \mathrm{~h}$ is larger than that between $\mathrm{COh}$ and T16h, while the difference between and the $\mathrm{COh}$ and $\mathrm{T} 2 \mathrm{~h}$ is the smallest of the three. That means, in L. lancifolium, transcript abundance changed dramatically at these key switches among the cold stress stages of $2 \mathrm{~h}$ to $16 \mathrm{~h}$ which the cold response genes could be induced and expressed largely, but we should not ignore the genes expression during the short-term of $0 \mathrm{~h}$ to $2 \mathrm{~h}$ cold treatment, because many important cold-stress response genes were up- and down-regulated in this period, they would earliest determine the plant to play instantaneous reflection and response to the cold stress. These findings suggested forecast that our analysis was capable of identifying cold stress response genes and therefore suitable for further investigation of the biological functions of these genes.

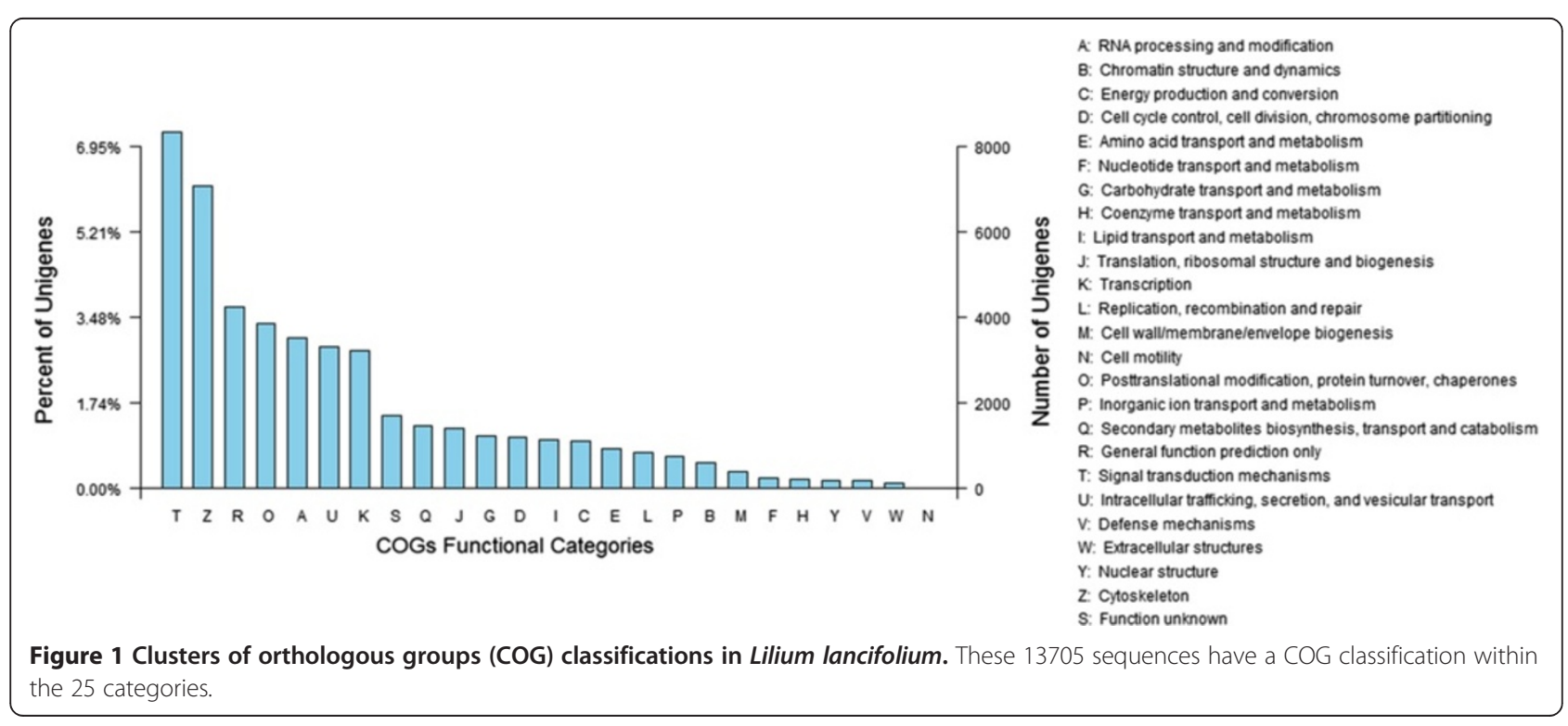




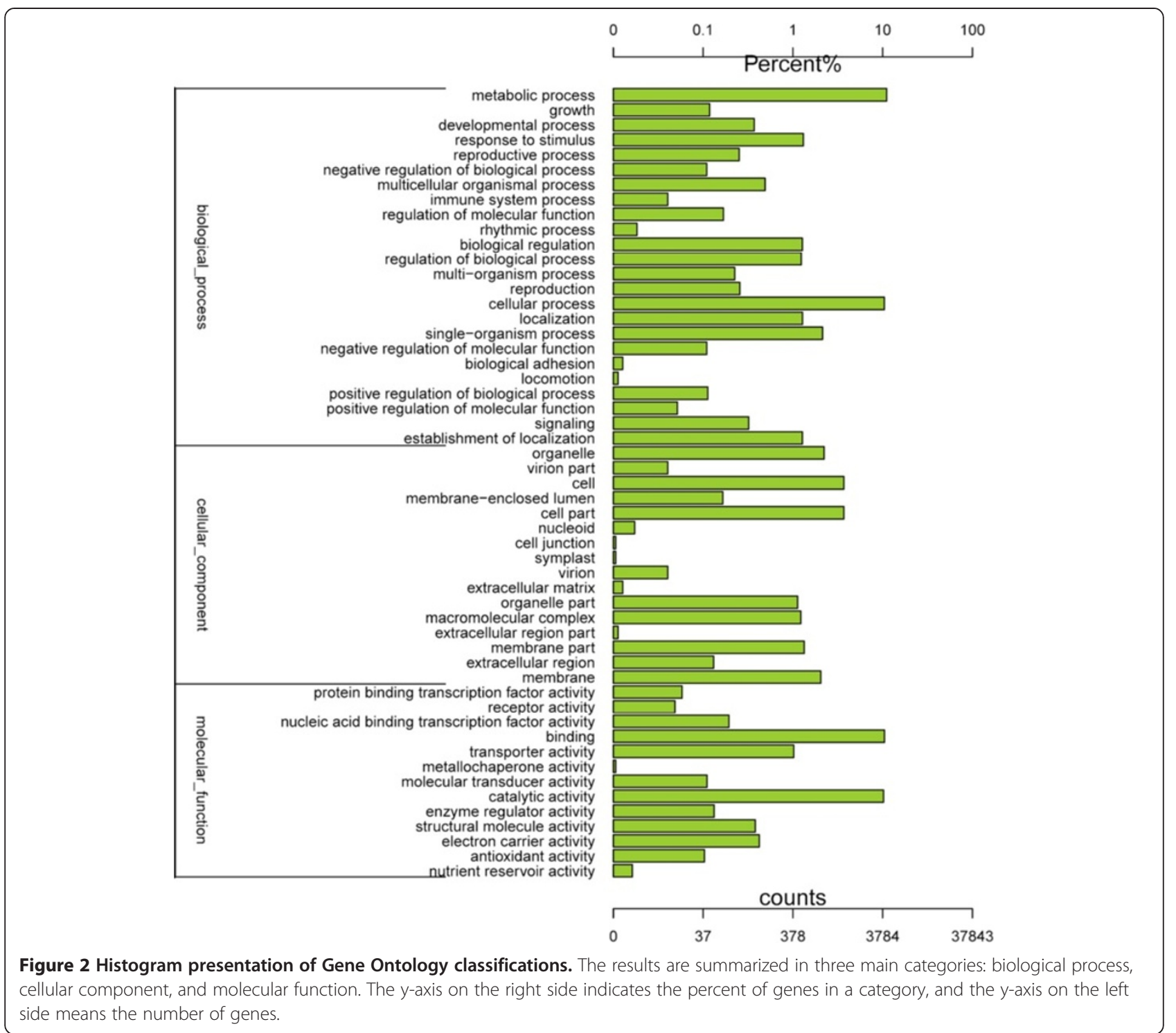

\section{SOM cluster analysis of gene expression}

To facilitate cluster analysis of gene expression, the expression profiles of the differentially expressed genes were determined by SOM cluster analysis based on the k-means method using Pearson's correlation distance. The total differential genes were divided into 12 groups based on their expression modulation with analysis of GO and KEGG pathway enrichment, representing the number of profiles as indicated by figure of merit analysis. Clusters were obtained by the k-means method using the gene expression profiles of the 1849 modulated genes. The most abundant group were Clusters 8 and 1, with 323 and 276 genes whose expression showed a positive slope during the T2h to T16h stage. The second most abundant group was Cluster 4, which contained 295 genes whose expression showed a negative slope from $\mathrm{COh}$ to $\mathrm{T} 2 \mathrm{~h}$. The functional category distribution frequency was then calculated for each cluster to identify differences in the distribution of genes among the three cold stress stages (Figure 4).

Clusters 2, 3, 6, 7, and 10 comprised 483 genes upregulation at $\mathrm{CT} 2 \mathrm{~h}$ but down-regulation in $\mathrm{CT} 16 \mathrm{~h}$, including different pathways as the 'energy production and conversion', 'inorganic ion transport and metabolism', 'extracellular structures', 'lipid transport and metabolism' and 'coenzyme transport and metabolism'genes. They indicate that cells accept the cold signal and instantly reflect using the ion transport and extracellular structure activities during the CT2h stage. In total, 802 genes upregulated in clusters $1,5,8$ and 9 in whole stages of cold response. They are specifically included in different pathways, such as 'carbohydrate transport and metabolism', 'amino acid transport and metabolism', 'translation, ribosomal structure and biogenesis' 'energy production 
Table 3 Categorization of Lilium Iancifolium unigenes to KEGG biochemical pathways

\begin{tabular}{|c|c|c|c|c|}
\hline KEGG categories & Mapped-ko & Unigene-NUM & Ratio of no. & Pathway-ID \\
\hline Metabolic pathways & 734 & 1784 & 11.23 & ko01100 \\
\hline Biosynthesis of secondary metabolites & 327 & 946 & 5.95 & ko01110 \\
\hline Microbial metabolism in diverse environments & 125 & 400 & 2.51 & ko01120 \\
\hline mRNA surveillance pathway & 48 & 256 & 1.61 & ko03015 \\
\hline Ribosome & 105 & 251 & 1.57 & ko03010 \\
\hline Pyrimidine metabolism & 63 & 250 & 1.57 & ko00240 \\
\hline Biosynthesis of amino acids & 95 & 237 & 1.49 & ko01230 \\
\hline Cell cycle & 50 & 233 & 1.46 & ko04110 \\
\hline Carbon metabolism & 71 & 229 & 1.44 & ko01200 \\
\hline Splice some & 87 & 223 & 1.40 & ko03040 \\
\hline Protein processing in endoplasmic reticulum & 71 & 208 & 1.31 & ko04141 \\
\hline RNA transport & 90 & 198 & 1.24 & ko03013 \\
\hline Starch and sucrose metabolism & 33 & 186 & 1.16 & ko00500 \\
\hline Purine metabolism & 77 & 169 & 1.06 & ko00230 \\
\hline Plant-pathogen interaction & 27 & 163 & 1.02 & ko04626 \\
\hline Plant hormone signal transduction & 38 & 158 & 0.97 & ko04075 \\
\hline Epstein-barr virus infection & 55 & 151 & 0.94 & ko05169 \\
\hline Oxidative phosphorylation & 70 & 150 & 0.94 & ko00190 \\
\hline Homologous recombination & 23 & 147 & 0.92 & ko03440 \\
\hline RNA degradation & 45 & 131 & 0.82 & ko03018 \\
\hline Ubiquitin mediated proteolysis & 55 & 128 & 0.81 & ko04120 \\
\hline Glycolysis/Gluconeogenesis & 30 & 128 & 0.81 & ko00010 \\
\hline Endocytosis & 38 & 127 & 0.79 & ko04144 \\
\hline Amino sugar and nucleotide sugar metabolism & 37 & 127 & 0.79 & ko00520 \\
\hline Basal transcription factors & 27 & 117 & 0.74 & ko03022 \\
\hline Phenylpropanoid biosynthesis & 16 & 109 & 0.68 & ko00940 \\
\hline Ribosome biogenesis in eukaryotes & 51 & 107 & 0.67 & ko03008 \\
\hline Oocyte meiosis & 29 & 106 & 0.66 & ko04114 \\
\hline Viral carcinogenesis & 38 & 104 & 0.66 & ko05203 \\
\hline Cell cycle-yeast & 47 & 102 & 0.64 & ko04111 \\
\hline Insulin signaling pathway & 19 & 102 & 0.64 & ko04910 \\
\hline HTLV-I infection & 40 & 101 & 0.63 & ko05166 \\
\hline Fatty acid metabolism & 10 & 100 & 0.62 & ko00071 \\
\hline Pyruvate metabolism & 28 & 96 & 0.60 & ko00620 \\
\hline Others & & 7874 & 11.22 & \\
\hline
\end{tabular}

and conversion', and 'signal transduction and mechanism' (Figure 4), suggesting that L. lancifolium initiates carbohydrate conversion and metabolism during $\mathrm{CT} 2 \mathrm{~h}$ to $16 \mathrm{~h}$.

Response of important transcription factors to cold stress Transcription factors (TF) play crucial roles not only as molecular switches for gene expression, but also as terminal points of signal transduction in the response to cold stress. At the $2 \mathrm{~h}$ and $16 \mathrm{~h}$ stages cold treatment for
L. lancifolium, the genes whose transcript abundance exhibited highly dynamic changes $\left(\mid \log _{2}(\right.$ ratio $) \mid \geq 4$, Figure 5) included genes for transcription factors (LlAP2/EREBP (KJ489026) transcription protein, LlNAC (KJ467622) domain transcription, LlERF2 transcription factor, LlBZIP transcription factor protein, LlMYBR (KJ467623) family domain class transcription factor), signal transport ( $A B C$ transporter, LlCalcium-transporting ATPase 4, LlZIP transporter, Zinc transporter, sugar transporter protein), stress kinases (LlCalcium-dependent protein kinase 1 


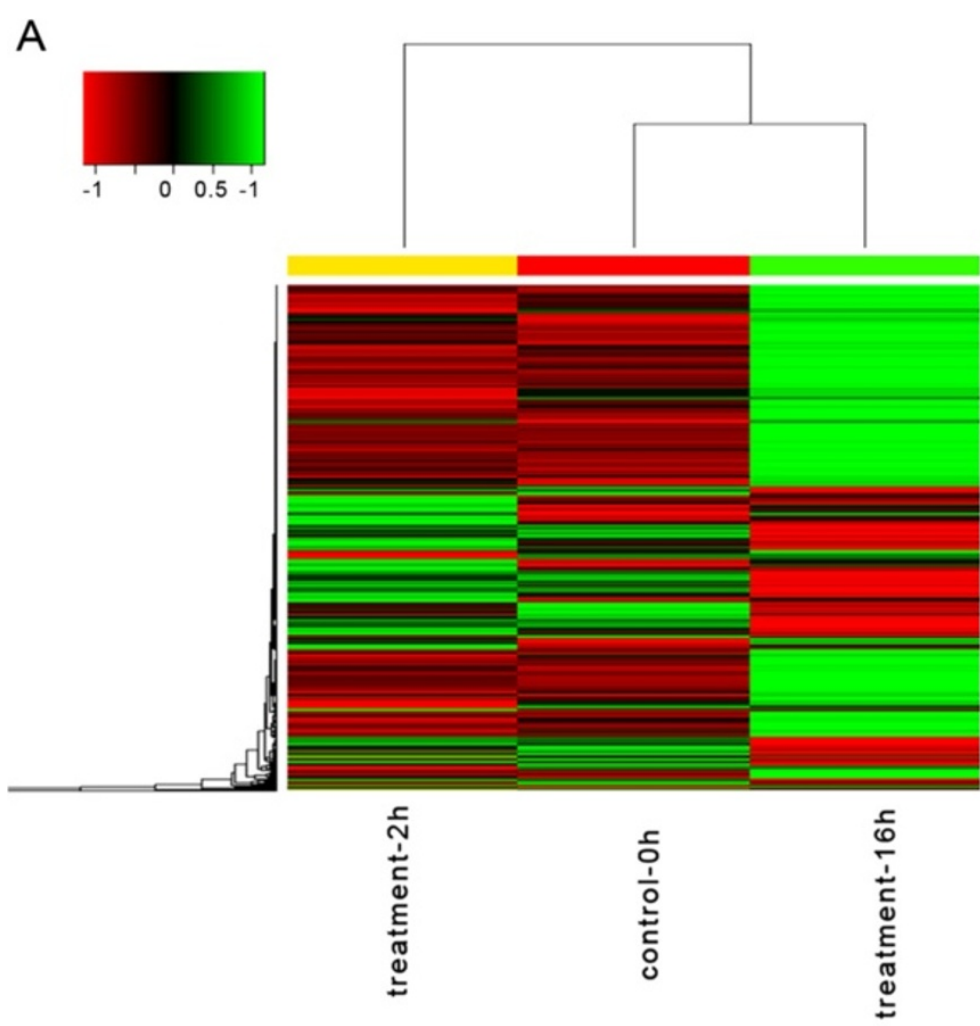

B

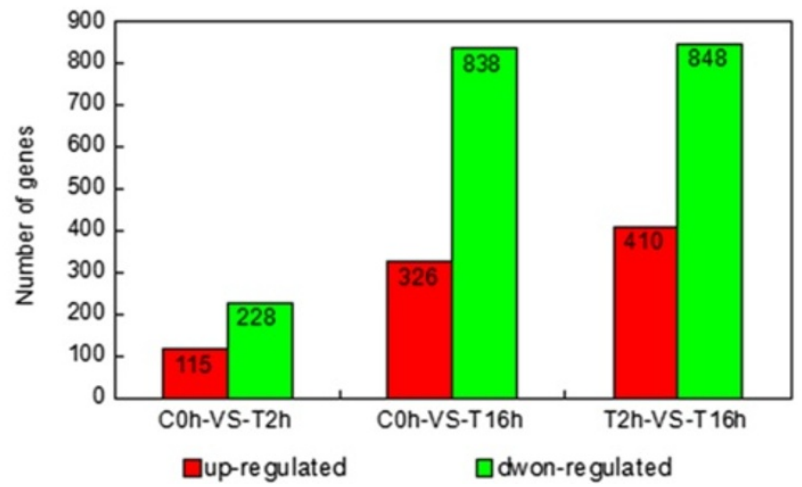

Figure 3 The expression of the gene changes among the different cold stress stages. A. The heat-map of the total differentially expressed genes (DEGs). Columns and rows in the heat maps represent samples and genes, respectively. Sample names are displayed below the heat maps. CK Results of controls, CT2h and 16h results of cold treatments. Color scale indicates fold changes of gene expression. A fold change of $\geq 1$ is shown in green (increased transcript abundance), a fold change of $\leq-1$ is shown in red (decreased transcript abundance), and no change is indicated in black. The results show that 1,028 transcripts were differentially expressed between the control and cold treatments $2 \mathrm{~h}$ and $16 \mathrm{~h}$. B. Changes in gene expression profile among the different cold stress stages. The number of up-regulated and down-regulated genes between COh-VS-T2h, COh-VS-T16h and T2h-VS-T16h are summarized. Between the COh and T2h Lilium lancifolium libraries, there are 115 genes up-regulated and 228 genes down-regulated, while there are 326 up-regulated genes and 828 down-regulated genes between the Coh and T16h Lilium lancifolium libraries, and 410 up-regulated genes 848 down-regulated genes between the C2h and T16h Lilium lancifolium libraries.

(KJ467621), LlCBL-interacting protein kinase 25, Serine/ threonine-protein kinase LlSAPK3, Putative LlsnRK/SAPK family protein kinase), low temperature induced-like proteins (DRE-binding protein LlDREB1/CBF (KJ467618), Cold-regulated LlCOR12 (KJ489025), Putative WRKY DNA-binding domain superfamily protein, Peptidyl-prolyl cis-trans isomerase, Copia LlLTR rider, Elicitor-inducible
LlLRR, Arachidonic acid-induced LIDEA1), and stressassociated compound proteins (Ll30S ribosomal protein, LlClass III homeobox-leucine zipper protein, LlHeat shock cognate $70 \mathrm{kDa}$ protein 2 (KJ467620), LlGPAT protein (KJ467617), Calcium-dependent calmodulinindependent protein (KJ467621); Figure 5, Additional file 1: Table S1). 

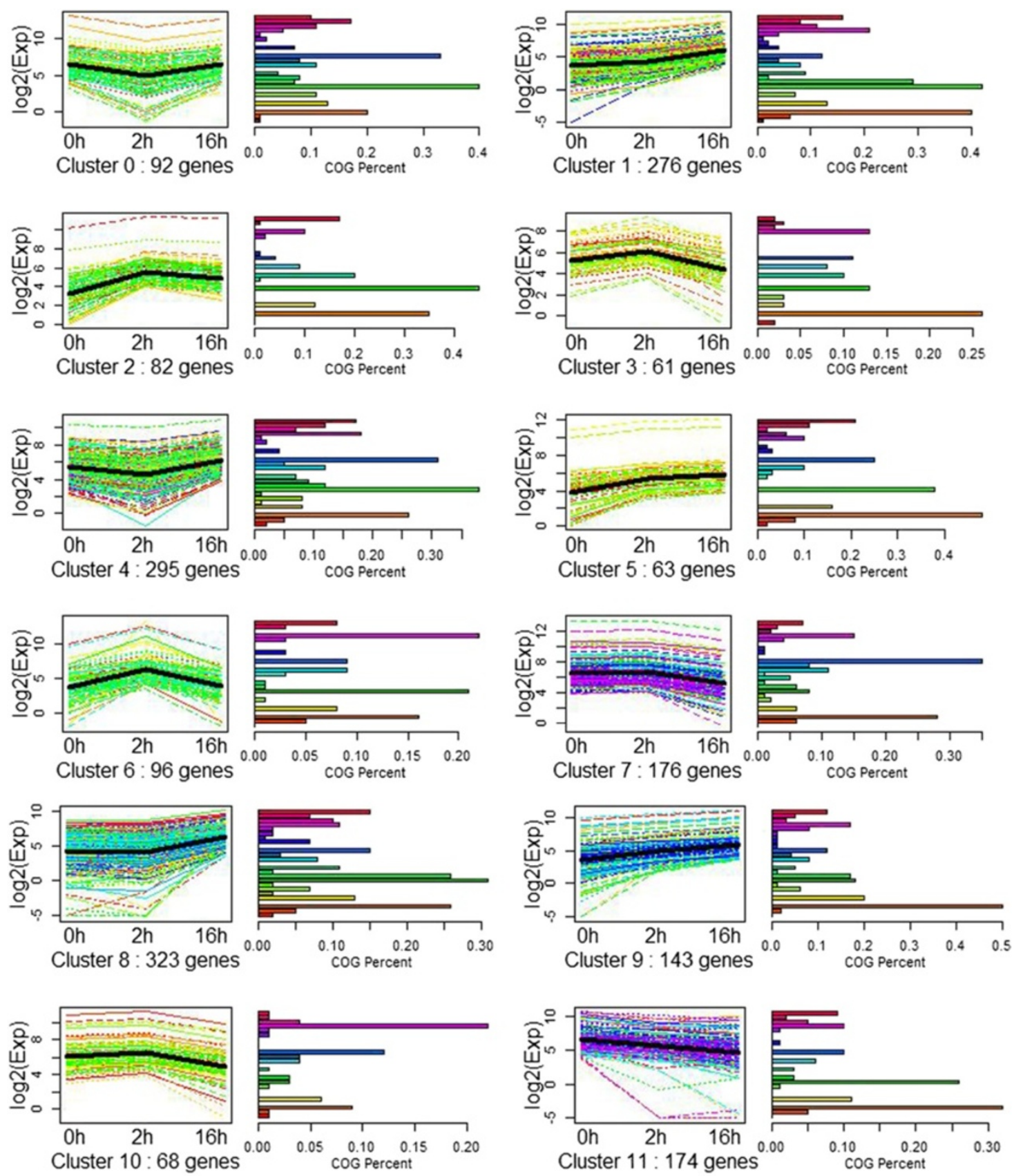

\begin{tabular}{|c|c|}
\hline $\begin{array}{l}\text { - A: RNA processing and modification } \\
\text { - B: Chromatin structure and dynamics } \\
\text { = C: Energy production and conversion } \\
\text { " D: Cell cycle control, cell division, chromosome partitioning } \\
\text { E: Amino acid transport and metabolism } \\
\text { - F: Nucleotide transport and metabolism } \\
\text { = G: Carbohydrate transport and metabolism } \\
\text { = H: Coenzyme transport and metabolism } \\
\text { - t: Lipid transport and metabolism } \\
\text { = J: Translation, ribosomal structure and biogenesis } \\
\text { - K: Transcription } \\
\text { " L: Replication, recombination and repair } \\
\text { " M: Cell walVmembrane/envelope biogenesis }\end{array}$ & $\begin{array}{l}\text { " N: Cell motilty } \\
\text { " O: Posttranslational modification, protein turnover, chaperones } \\
\text { - P: Inorganic ion transport and metabolism } \\
\text { " Q: Secondary metaboltes biosynthesis, transport and catabolism } \\
\text { " R: General function prediction only } \\
\text { - T: Signal transduction mechanisms } \\
\text { " U: Intracellular trafficking, secretion, and vesicular transport } \\
\text { - V: Defense mechanisms } \\
\text { - W: Extracellular structures } \\
\text { " Y: Nuclear structure } \\
\text { - Z: Cytoskeleton } \\
\text { " S: Function unknown }\end{array}$ \\
\hline
\end{tabular}

Figure 4 (See legend on next page.) 
(See figure on previous page.)

Figure 4 SOM cluster analysis of gene expression in the 12 different patterns. Clusters were obtained by the k-means method on the gene expression profiles of the 1849 modulated genes. The most abundant group is Cluster 8 and 1, with 323 and 276 genes whose expressions show positive slopes during stage of T2h to T16h embryogenesis. The second abundant group is Cluster 4, which contained 295 genes whose expression shows a negative slope from COh to T2h.

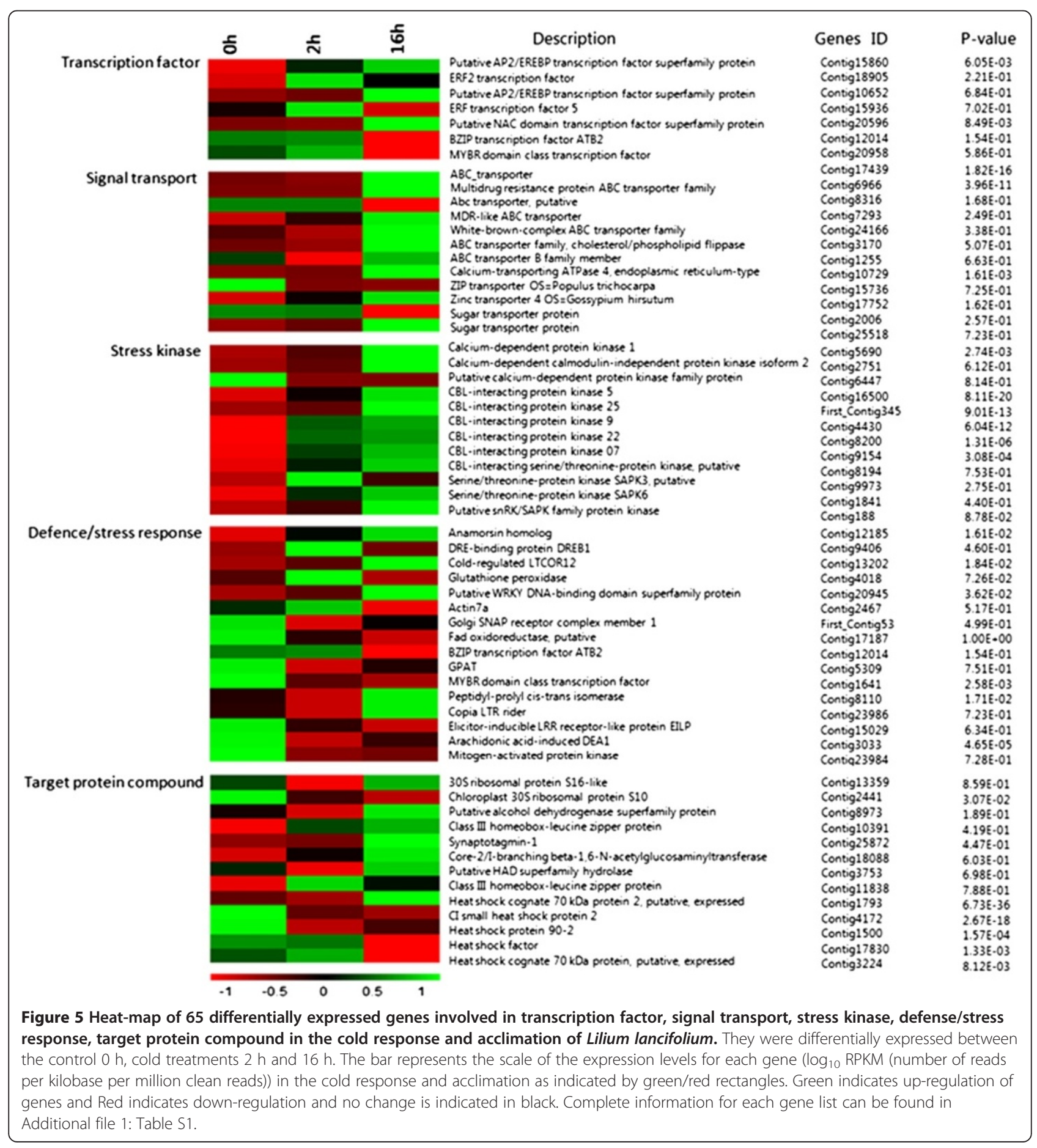


Therefore, the changes in the expression patterns of distinct transcripts suggest a requirement for different developmental events when L. lancifolium is under cold stress. For example, seven transcription factors preferentially expressed and 12 signal transport transcripts accumulated to a higher level at $2 \mathrm{~h}$ than $16 \mathrm{~h}$, which indicated that transcription factors reacted more actively to cold in the initial short-term response rather than the long-term cold acclimation (Figure 5, Additional file 1: Table S1). Transcription factors (TF) act as switches and terminal points of signal transduction in a stress-specific fashion in the response to cold stress. In addition, 12 stress kinase protein genes and 13 stress-associated compound protein genes showed the highest accumulation in the cold-treatment $16 \mathrm{~h}$ stages which was expected, as expected for cold resistance. Interestingly, the 16 genes related to low temperature induced-like proteins showed different trends. Three of them showed higher expression in $\mathrm{CT} 2 \mathrm{~h}$ and then decreased in CT16h, such as DRE-binding protein LIDREB1, LlCBLinteracting protein kinase, Glutathione peroxidase and LlBZIP transcription factor ATB2, and five of them reached the highest expression value at $16 \mathrm{~h}$, including Cold-regulated LlCOR12, LlWRKY DNA-binding domain superfamily protein, Copia LlLTR rider and Peptidyl-prolyl cis-trans isomerase; the rest of them were down-regulated from $0 \mathrm{~h}$ to $16 \mathrm{~h}$ (Figure 5, Additional file 1: Table S1). All of these results may contribute to identifying the signaling development networks in response in the cold reponse of L. lancifolium.

\section{Verification of the gene expression profiles by qRT-PCR}

To further verify the expression profiles of the genes in our Illumina sequencing analyses, we selected 10 DEGs for qRT-PCR using samples of leaves, stems, roots and bulbs originally used for RNA-Seq, all of which are known to be related to cold stress, including the genes encoding LlAP2 (putative AP2/EREBP transcription factor, KJ489026), LlLEA (late embryogenesis abundant protein, KJ489024), LlCIS (peptidyl-prolyl cis-trans isomerase, KJ467624), LIDREB1 (dehydration-responsive element binding, KJ467618), LlHOT (heat shock protein, KJ467620), LlCOR12 (cold-regulated LTCOR12, KJ489025), LIMYBR (MYBR domain class transcription factor, KJ467623), LlNAC1 (NAC domain protein, KJ467622), and LlCDPK (calcium-dependent protein kinase, KJ467621), LlGPAT (KJ467617). The Ct values of the LlTIP1 rRNA in all samples ranged from 24.0 to 26.0. All 10 transcripts showed the same expression pattern as the in silico differential analysis results from high-throughput sequencing. We have provided GenBank accession numbers for our gene nucleotide sequences in Table 4 and Additional file 2: Txt S3.
These genes were selected for their key roles in regulating stress signal transcription, cold responses, and cold acclimation. The results presented in Figure 6A and $6 \mathrm{~B}$ showed that the expression levels of six genes were higher during CT2h to CT16h than in the other stages, including LlCIS, LlMYBR, LlGPAT, LlCOR12, LlDREB1 and $L l C D P K$, indicating their signal transduction and transcriptions reaction after receiving the cold signal. Four genes, encoding LlAP2, LlLEA, LINAC1 and LlHOT, were more highly expressed between $24 \mathrm{~h}$ and $48 \mathrm{~h}$ than in the initial stages, and showed higher expression values than the former six genes (Figure 6A B), demonstrating that these genes may react slowly after the transcription factors and are related to cold acclimation. Also, the expression levels of the LILEA, LINAC1, LlCDPK and LIDREB1 genes were highest in bulb at $2 \mathrm{~h}$ and $16 \mathrm{~h}$ than in the other tissues, including roots and stems and reached a higher value in the stem CT2h sample. The $L l C D P K$ genes were remarkably highly expressed in roots at $16 \mathrm{~h}$ (Figure $6 \mathrm{C}, \mathrm{D}$ ). We predicted that the bulb has played an important role in for the L. lancifolium to resistance and adaptation to cold stress. These results indicated that there was a close correlation between the expression changes (fold difference) measured by RNA-Seq and those by qRT-PCR (Table 4 ).

\section{Soluble protein, starch, soluble sugar and Malondialdehyde concentration}

To elucidate the mechanism underlying the cold response, it is important to determine how plants alter gene expression in response to this biological process. The levels of two measured soluble proteins increased sharply and rapidly following cold exposure of L. lancifolium and Oriental hybrid leaves, but the soluble protein levels of L. lancifolium declined more sharply and faster than those of the Oriental hybrids from $2 \mathrm{~d}$ to $14 \mathrm{~d}$ of cold exposure, and the totals of the two varieties showed significant differences that reached a maximum at $24 \mathrm{~h}$ (Figure 7A, Additional file 1: Table S2). On an area basis, starch tests showed that L. lancifolium leaves had increasing starch content between $1 \mathrm{~h}$ to $48 \mathrm{~h}$ of cold treatment, which rapidly fell during subsequent cold stress; although unlike L. lancifolium, the cold development slowly and incrementally increased the starch content of the Oriental hybrids leaves (Figure 7B, Additional file 1: Table S2). Soluble sugar levels also decreased, with linear regression significant on a CT basis, and exhibited a greater decrease in L. lancifolium than in the Oriental hybrids when measured at $4^{\circ} \mathrm{C}$ (Figure $7 \mathrm{C}$, Additional file 1: Table S2); however, the data suggested a peak at 16-24 h. Total nonstructural carbohydrates (TNCs) responded non-linearly, because of the influence of starch and glucose. Both in L. lancifolium and Oriental hybrid leaves, cold development increased MDA levels 
Table 4 Primers used in real-time quantitative PCR of Lilium lancifolium (RT-qPCR)

\begin{tabular}{|c|c|c|c|c|c|c|}
\hline Unigene Id & $\begin{array}{l}\text { Gene } \\
\text { name }\end{array}$ & Annotation & Forward primer sequence $\left(5^{\prime}-3^{\prime}\right)$ & Reverse primer sequence $\left(5^{\prime}-3^{\prime}\right)$ & $\begin{array}{l}\text { Correlation } \\
\text { between RNA-Seq } \\
\text { and qRT-PCR }\left(r^{2}\right)\end{array}$ & $\begin{array}{l}\text { GenBank } \\
\text { accession } \\
\text { numbers }\end{array}$ \\
\hline Contig15860 & LIAP2 & $\begin{array}{l}\text { Putative AP2/ } \\
\text { EREBP } \\
\text { transcription } \\
\text { factor }\end{array}$ & CCGCCCTCTTCAATCTCATC & TATCTGGCTCGGCTCCTAC & 0.98 & KJ489026 \\
\hline Contig 18777 & LILEA & LEA-like protein & AAGATGTTCCTCCTCGTGTTG & GATGTTGGTCCTCGCCTTC & 0.99 & KJ489024 \\
\hline Contig11020 & LICIS & $\begin{array}{l}\text { Peptidyl-prolyl } \\
\text { cis-trans } \\
\text { isomerase }\end{array}$ & TTGTTCCTTCCACCGCATTA & AAAGCCTTCATCCTCAAACTTAGAC & 0.95 & KJ467624 \\
\hline Contig9406 & LIDREB1 & $\begin{array}{l}\text { DRE-binding } \\
\text { protein }\end{array}$ & AAATCCGCCTCCCCAAGAA & AGTTGAGCCGAGCGAAGT & 0.98 & KJ467618 \\
\hline Contig1500 & LIHOT & $\begin{array}{l}\text { Heat shock } \\
\text { protein }\end{array}$ & ATGATTGGGAGGAGCACTTG & GAAGACACGGCGGACATAA & 0.97 & KJ467620 \\
\hline Contig13202 & LICOR12 & $\begin{array}{l}\text { Cold-regulated } \\
\text { LTCOR12 }\end{array}$ & CGGACACAACTTGACTCTTACC & CTTGCTATGCCTCGCTGAC & 0.94 & KJ489025 \\
\hline Contig1641 & LIMYBR & $\begin{array}{l}\text { MYBR domain } \\
\text { class } \\
\text { transcription } \\
\text { factor }\end{array}$ & TTCCTCAGTCCACGCTATCC & GCCGTTGCCTAACTACTTGTC & 0.98 & KJ467623 \\
\hline Contig25399 & LINAC1 & $\begin{array}{l}\text { NAC domain } \\
\text { protein }\end{array}$ & GGTIAGAGGGAGGTTGGAGAAG & CGACGACACTGGCTCATCA & 0.94 & KJ467622 \\
\hline Contig22048 & LICDPK & $\begin{array}{l}\text { Calcium- } \\
\text { dependent } \\
\text { protein kinase }\end{array}$ & GTCGTGCTCCAATTACCAGAAG & CAAGAGGAACAACATCACCAGAC & 0.94 & KJ467621 \\
\hline \multirow[t]{2}{*}{ Contig5309 } & LIGPAT & GPAT & TGCAAAGTGGAAATCCTAATGCGAG & AGGATCTGCTTCCGTCTGATGGTTT & 0.97 & KJ467617 \\
\hline & LITIP1 & Reference gene & CGAAGCCAGAAACGGAGAAGAAT & GGGTAGGGTGGATTGGGAAGA & & \\
\hline
\end{tabular}

relative to the cold response from $1 \mathrm{~h}$ to $16 \mathrm{~h}$, whereas, MAD content in L. lancifolium leaves declined significantly with increasing cold exposure duration at $4^{\circ} \mathrm{C}$ in contrast to the increase in the Oriental hybrids during the same stage (Figure 7D, Additional file 1: Table S2). The L. lancifolium leaves responded more strongly than Oriental hybrid leaves during the cold treatment.

\section{Membrane systems and cellular osmoprotectant}

Membrane systems, which are known to be the primary site of freezing injury in plants, suffer multiple forms of damage caused by freeze-induced cellular dehydration [22]. During cold acclimation, plants experience improved cold tolerance with increased cellular metabolic activity, and increased concentrations of unsaturated fatty acids and phospholipids [23]. Correspondingly, we identified a total of 1153 genes (4.46\%) involved in 'Lipid transport and metabolism' and 3333 genes (7.01\%) involved in 'Intracellular trafficking, secretion, and vesicular transport' according to the COG classification. Furthermore, according to the metabolic pathway enrichment analysis, the 'biosynthesis of unsaturated fatty acids' (ko01040), 'fatty acid elongation' (ko00062) and 'regulation of actin cytoskeleton' (ko04810) pathways were all involved in lipid metabolism. Increases in the biosynthesis of unsaturated fatty acids improve cold defense and prevent damage caused by low temperatures [24]. Thirty genes in this process showed significant regulation of their transcripts after cold stress. For example, two chloroplast omega-3 fatty acid desaturase genes (Contig10730_All, Contig17187_All) were up-regulated by 0.38 to 1.15 -fold. The FAD3 gene in L. lancifolium encodes a chloroplast membrane-associated omega-3 fatty acid desaturase, which contributes to freezing tolerance by altering the lipid composition [25]. In our present study, the analysis of the structural characteristics of L. lancifolium leaf cells showed that leaf thickness doubles compared with room-temperature controls during cold treatment. This thickening is a result of palisade cell length elongation and an enlargement of the intercellular spaces caused by a more loosely packed spongy parenchyma matrix [26]. The intercellular spacing and spongy parenchyma packing were homoplastically altered following the cold treatment, they were close together at $16 \mathrm{~h}$, but the palisade cell length and spacing increased prior to $48 \mathrm{~h}$, and was most altered after $7 \mathrm{~d}$ of treatments (Figure 8).

Proline, one of the most effective organic osmolytes, participates in responses to various environmental stresses [27]. Moreover, according to the metabolic pathway enrichment analysis, 'proline metabolism' (ko00330) involved 69 genes that regulate and relieve the osmotic stress 

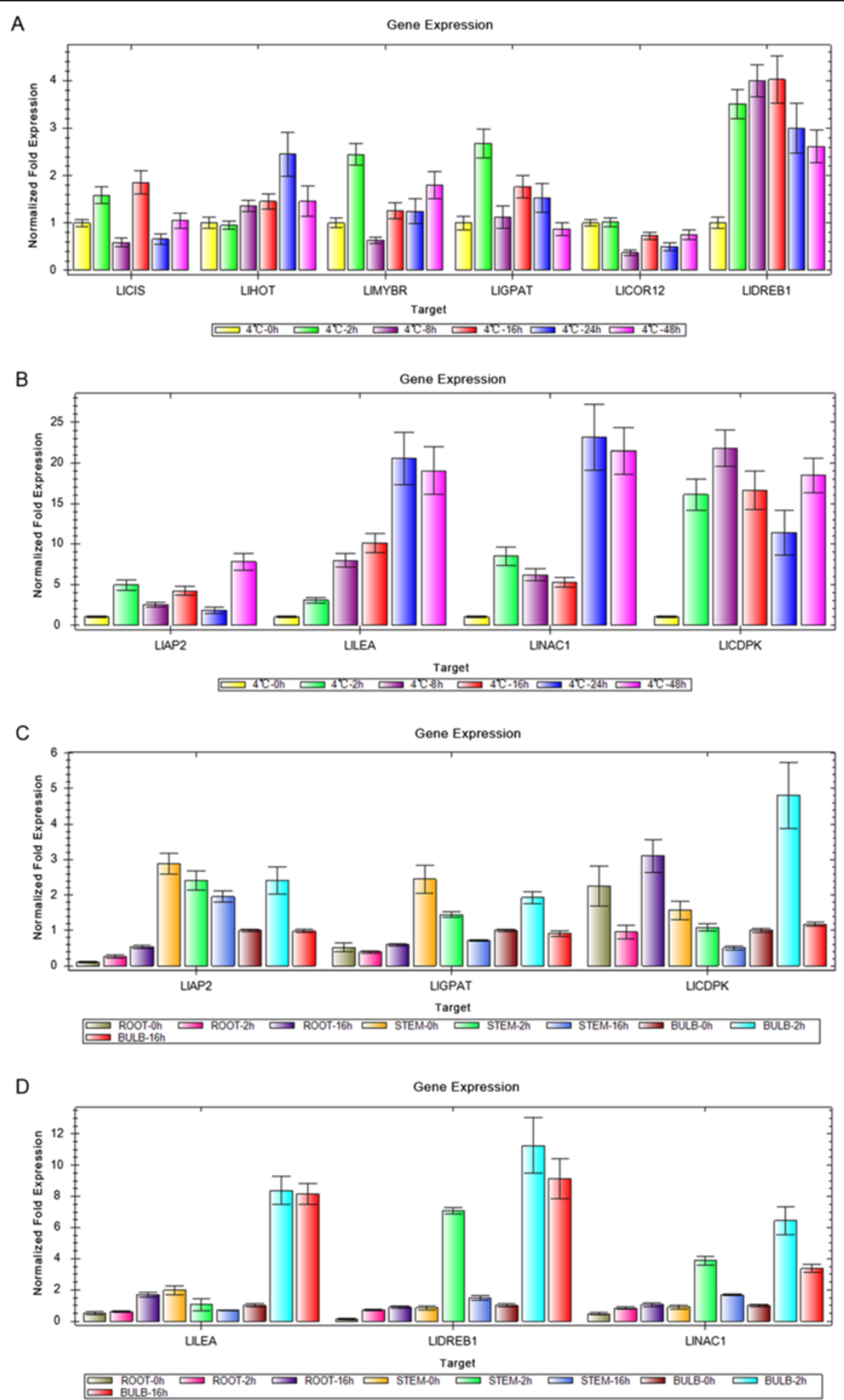

Figure 6 The expression profiles of 10 transcripts in Lilium lancifolium by the quantitative reverse transcription polymerase chain reaction (qRT-PCR). The Figures $\mathbf{A}$ and $\mathbf{B}$ indicated the expression of ten genes from their leaves; the Figures $\mathbf{C}$ and $\mathbf{D}$ implied the expression of six genes from their stems, roots and stems. The $y$-axes show normalized fold expression levels determined by qRT-PCR. 


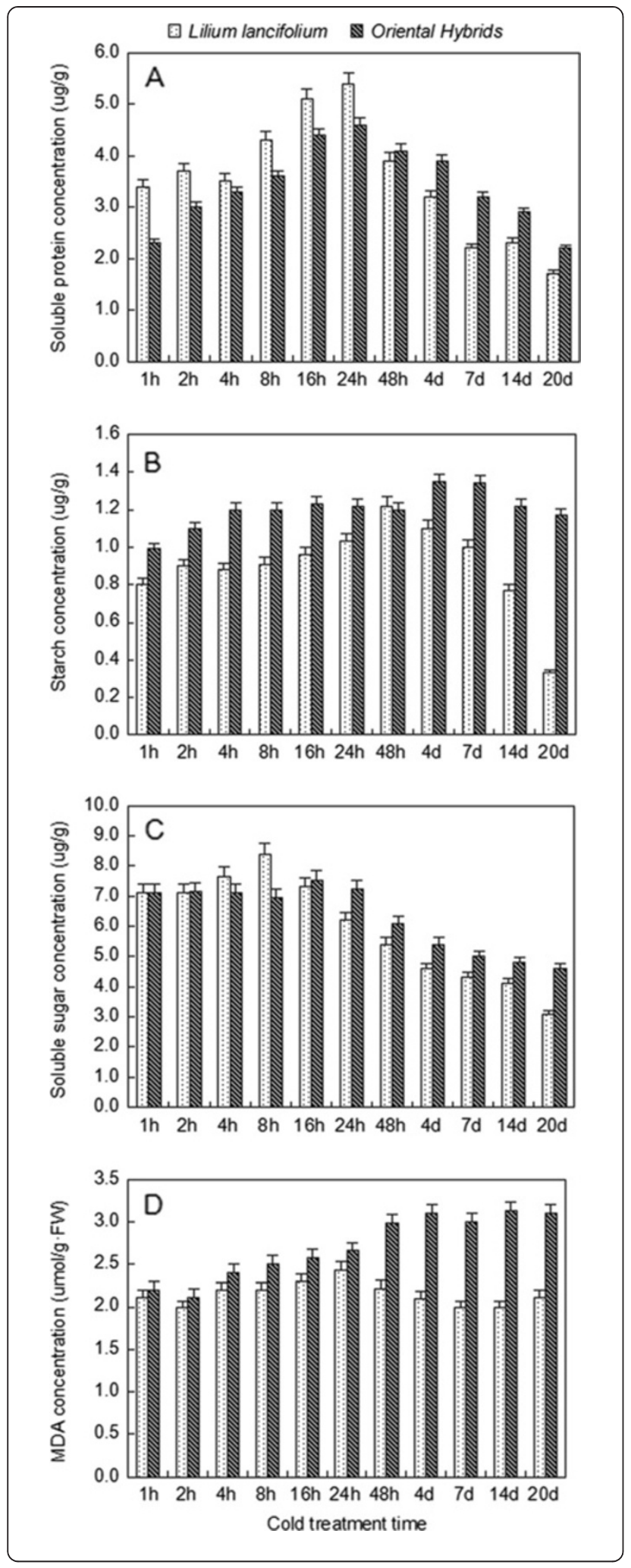

Figure 7 Soluble sugar, starch, soluble protein and MDA concentration per unit $4^{\circ} \mathrm{C}$ cold treatment (CT) of Lilium lancifolium and Oriental hybrids' leaves. A. Two cultivars' soluble protein content in different stages under $4^{\circ} \mathrm{C}$ cold treatments $\mathbf{B}$. Two cultivars' starch content in different stages under $4^{\circ} \mathrm{C}$ cold treatments $\mathbf{C}$. Two cultivars' soluble sugar content in different stages under $4^{\circ} \mathrm{C}$ cold treatments $\mathbf{D}$. Two cultivars' MDA content in different stages under $4^{\circ} \mathrm{C}$ cold treatments Black squares: Lilium lancifolium; grey squares: Oriental hybrids'fructose; Bars refer to standard errors.

caused by cold-induced dehydration. Two genes (Contig7616, Contig7617) for delta1-pyrroline-5-carboxylate synthetase (P5CS) were significantly up-regulated by 0.88 and 1.29-fold after cold stress, respectively. As a key enzyme in proline synthesis, DREB1 participates in the cold-stress response and shows high expression, which promotes the synthesis of proline for cold tolerance [28]. The accumulation of sucrose and other simple sugars also contributes to the stabilization of membranes, as these molecules protect membranes against freezeinduced damage in vitro. Five $L E A$ (late embryogenesis abundant)-related genes (Contig24352_All, Contig19954 _ All, Contig17189_ALL, Contig26956, Contig18777) were also differentially expressed with fold changes in their expression ranging from 0.23 to 1.30 -fold. The LEA protein functions as an antioxidant, as well as a membrane and protein stabilizer, during cold stress [29]. Our studies have analogously suggested that the electrical conductivity of $L$. lancifolium leaves decreases at $2 \mathrm{~h}$ after but increases at $12 \mathrm{~h}$, and then keeps a positive slope until $24 \mathrm{~h}$ cold treatment It is reasonable to infer that hydrophilic and LEA polypeptides stabilize membranes against freezing-induced injury in L. lancifolium [30]. Proline levels further enhanced the electrical conductivity of L. lancifolium leaves in different stages under $4^{\circ} \mathrm{C}$ cold treatment (Figure 9). The electrical conductivity dropped at $2 \mathrm{~h}$, but kept gradually increasing with prolonged cold treatment. It can be inferred that the accumulation and effects of Proline may contribute to a remarkable control of cellular electrical conductivity, which reflects the destruction of cell wall and cytoplasm motivation, furthermore regulating outstanding cold tolerance for L. lancifolium (Figure 9).

\section{Discussion}

Illumina paired-end sequencing, assembly, and functional annotation

Transcriptome analysis is important for elucidating the molecular constituents of cells and tissues and interpreting the functional elements of the genome [31]. In our study of the transcriptome of L. lancifolium $(3 \mathrm{n}=27)$, we sampled the pooled transcriptomes of leaves using Illumina paired-end sequencing technology to generate a large-scale EST database. Approximately 10.7 GB of data was generated and assembled into 37,843 unigenes. This 

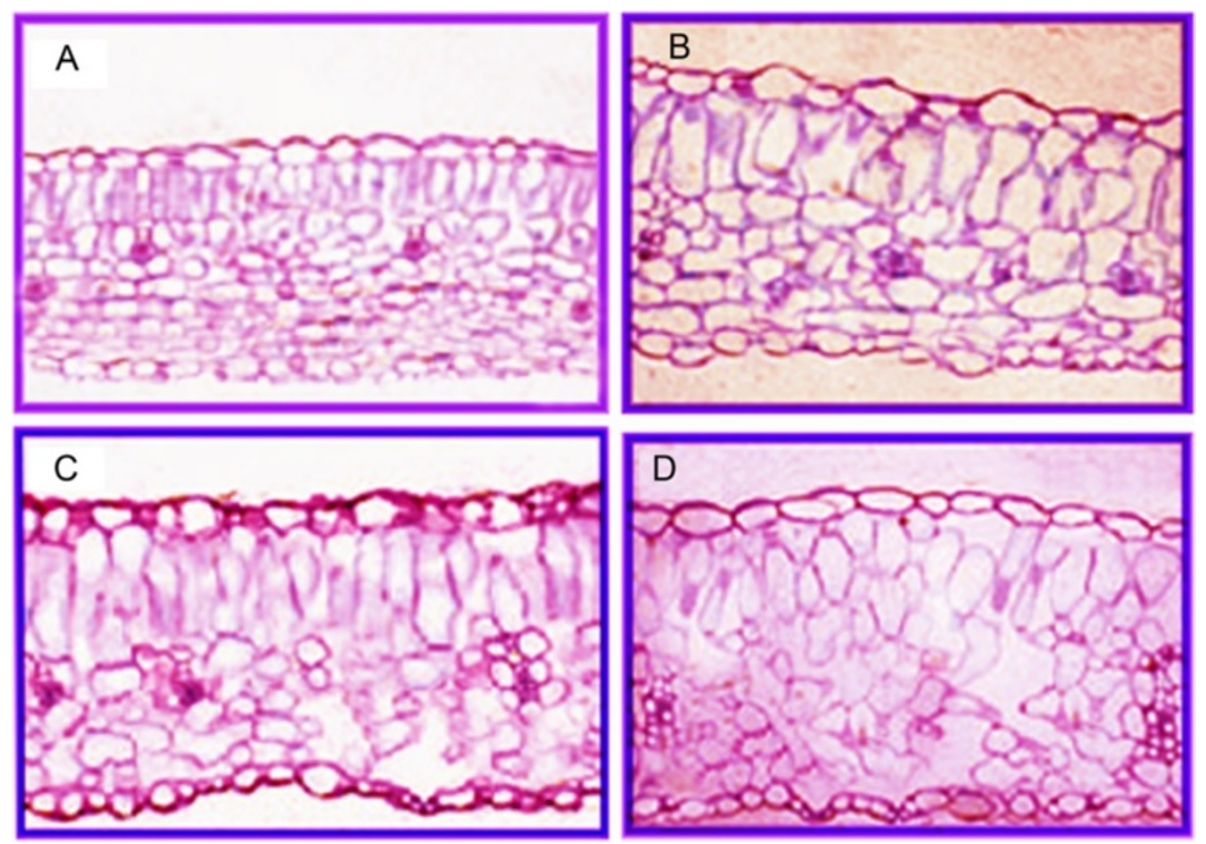

Figure 8 Lilium lancifolium leaf structures in different stages under $4^{\circ} \mathrm{C}$ cold treatments. A. Controlled $0 \mathrm{~h}$ (grow up in room-temperature) leaf structure; B. cold treatment $16 \mathrm{~h}$ leaf structure; C. cold treatment $48 \mathrm{~h}$ leaf structure; $\mathbf{D}$. cold treatment 7 days leaf structure.

large number of reads with paired-end information produced much longer unigenes (mean, $973 \mathrm{bp}$ ) than those in other lily studies. This increased coverage depth of the transcriptome facilitated de novo assembly, enhanced sequencing accuracy, and avoided possible contamination. Of the $37,843 \mathrm{~L}$. lancifolium unigenes, 18,736 (49.30\%) had homologs in the Swiss-Prot database. More importantly, we were able to assign a number of these unigenes to a wide range of GO categories and COG classifications (Figures 1 and 2), indicating that diverse transcripts are involved in the cold response and which are represented in the sequence data of this species, and also reflecting the complexity of differential low temperature signal transcription in L. lancifolium.

Furthermore, during cold responses and tolerance, plants receive low temperature signals and initiate a defense mechanism, including physical structure adaptations (changes in lipid composition and extracellular metabolic activity), increases in intercellular osmoprotectants (such as soluble sugars, proline and betaine), and up-regulated synthesis of anti-oxidants (superoxide dismutase, pathogen defense, catalase and ascorbic acid reductase), enabling restoration of the balance of biosynthesis and carbohydrate metabolism and enhancing survival in cold environments [32]. Interestingly, part of the expression patterns of a large number of genes during cold stress stimuli and transcription factors have been detected using transcriptome sequencing and microarray technologies. Most representative unigenes were annotated to specific pathways, such as metabolic pathways, biosynthesis of secondary metabolites, microbial metabolism in diverse environments, the mRNA surveillance pathway, ribosomes, pyrimidine metabolism, biosynthesis of amino acids, the cell cycle, carbon metabolism and plant hormone signal transduction using the KEGG databases (Table 3), leading us to conclude that most of the genes we identified are involved in the cold response and signaling regulation.

\section{Carbohydrate metabolism}

A principal factor in carbohydrate metabolism under stress conditions is regulation of the balance between biosynthesis and breakdown of proteins. Proteolysis plays a dynamic and vital role in the regulation of different metabolic processes, and in the cell's response to environmental stimuli. It controls metabolic fluxes by regulating the levels of key rate-limiting enzymes, while also irreversibly irreversibly polypeptides into soluble sugars that may

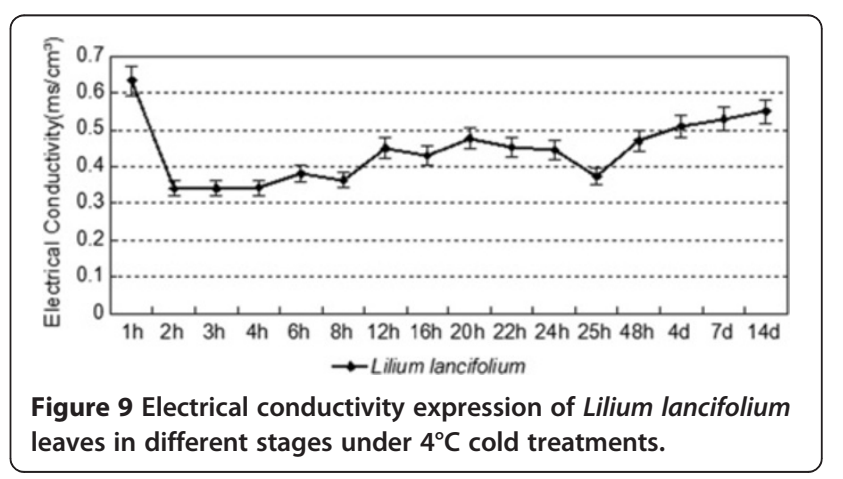


interfere with these pathways. Much of this directed protein turnover is performed by proteases that require ATP and the Clp protease is one of the best characterized to date [33]. Through our KEGG analysis, we discovered 186 regulated genes in the 'Starch and sucrose metabolism' pathway and 208 regulated genes in the 'Protein processing in endoplasmic reticulum' pathway (Table 3). Moreover, the protein of L. lancifolium increased with the rapid cold stimulus and then degraded into sugars with a faster reduction trend than the Oriental hybrids during the cold treatment. This demonstrated that L. lancifolium could adapt to $4^{\circ} \mathrm{C}$ cold treatment better than the Oriental hybrids, by accumulating protein and converting it into sugar; increasing the cell liquid concentration and reducing the freezing point, so as to prevent the frost damage (Figure 7A, Additional file 1: Table S2). This supports the fact that $L$. lancifolium can acclimate to a cold environment and elicit a series of physiological and biochemical responses to low temperature, such as the transformation and combination of soluble sugars (Figure $7 \mathrm{C}$, Additional file 1: Table S2). The reason for the difference is that Oriental hybrids grow in slightly lower latitudes than L. lancifolium and their optimum growth temperature is $25^{\circ} \mathrm{C}$. Interestingly, in the L. lancifolium transcriptome, we found related proteolytic enzymes, including an ATPdependent Clp protease proteolytic subunit, with a fold change of 0.94 (down-regulation) from $0 \mathrm{~h}$ to $2 \mathrm{~h}$ but a fold change of 1.28 (up-regulation) during $2 \mathrm{~h}$ to $16 \mathrm{~h}$ (Contig24056_All), a Clp-like energy-dependent protease, which showed a 1.56 fold change (Contig1979_All), and also Sucrose phosphate synthase and Glucose-6-phosphate 1-dehydrogenase, which were up-regulated with 1.67 and 1.12 fold changes, respectively (Contig7878_All, Contig3504_All). Overall, the data suggested that transfer of L. lancifolium leaf soluble protein is crucial for determining soluble synthesis, and induces better cold resistance than Oriental hybrid leaves, which cannot respond and acclimate to low temperature (Figure 7C, Additional file 1: Table S2).

The enzymes Alpha-amylase, $\beta$-amylase and Isoamylase degrade starch to soluble sugar, the further conversion of which leads to increased maltose, glucose, fructose and sucrose levels [34]. The effects of CT and cold duration on the expression of alpha-amylase, $\beta$ amylase, and isoamylase in L. lancifolium were shown by Illumina sequencing, with 2.25 -fold up-regulation from $0 \mathrm{~h}$ to $2 \mathrm{~h}$ and 0.93 -fold down-regulation from $2 \mathrm{~h}$ to $16 \mathrm{~h}, 0.73$-fold up-regulation from $0 \mathrm{~h}$ to $2 \mathrm{~h}$; and 1.78fold up-regulation from $0 \mathrm{~h}$ to $2 \mathrm{~h}$ and 0.70 -fold downregulation, from $2 \mathrm{~h}$ to $16 \mathrm{~h}$, respectively (Contig5436_all, First_Contig433_all, Contig13233_all). Thus, it is unsurprising that a subset of starch hydrolysis enzyme genes display a highly specific, largely up-regulation response between $0 \mathrm{~h}$ to $2 \mathrm{~h}$ of $4^{\circ} \mathrm{C}$ cold stress, because hydrolysis enzymes affect the degradation of carbohydrate during the early period of cold stress [35]. On an area basis, starch tests showed that the starch content of L. lancifolium leaves had increased between the $1 \mathrm{~h}$ to $48 \mathrm{~h}$ of cold treatment, and fell during subsequent cold stress as it was degraded into soluble sugar. The limited starch degradation of Oriental hybrid leaves indicated its poor ability to resist and adapt to cold exposure (Figure 7B, Additional file 1: Table S2). In L. lancifolium, we detected that the expression of 47 soluble sugar synthase DEGs out of 1326 different enzyme DEGs was significantly and markedly induced by $2 \mathrm{~h}$ and $16 \mathrm{~h}$ cold stress, including five up-regulated and 19 down-regulated glucose synthase genes, two up-regulated and four downregulated fructose synthase genes and three up-regulated and 14 down-regulated sucrose synthase genes. Thus, exposure to $4^{\circ} \mathrm{C}$ cold treatment clearly favored starch degradation, which would result in increased accumulation of soluble sugars. In addition to the effects of chilling on $\beta$-amylase transcript levels, we found that $\mathrm{CT}$ increased the expression levels of a specific Sucrosephosphate synthase7 (SPS7) transcript (First_Contig33) by 0.73 -fold. Because SPS1 catalyzes sucrose synthesis, it is possible that increased accumulation of sucrose in L. lancifolium may contribute to cold tolerance (Figure 7B, C, Additional file 1: Table S2).

MDA (malondialdehyde) reflects the ability of plants to resist and acclimate to abiotic stress [36]. In L. lancifolium under $4^{\circ} \mathrm{C}$ cold treatment, MDA showed no obvious change during the early cold period but declined during the $48 \mathrm{~h}$ to $20 \mathrm{~d}$ period, which demonstrated the better cold resistance characteristics of in L. lancifolium compared with the Oriental hybrid (Figure 7D, Additional file 1: Table S2). To some extent, it is possible that MDA is related with proline, and the up-regulation of the delta1pyrroline-5-carboxylate synthetase (P5CS, Contig7616_all, 1.29 fold) would enhance proline concentration to strengthen the plant's osmotic adjustment ability, resulting in the mitigation of cellulose peroxidation.

\section{Signaling pathways}

In endocrine signaling, hormones act on distant target cells in paracrine signaling, a molecule released from a cell acts on nearby targets and in autocrine signaling, a cell produces a signaling molecule to which it also responds [36]. The binding of most signaling molecules to their receptors initiates a series of intracellular reactions that regulate virtually all aspects of cell behavior, including metabolism, movement, proliferation, survival, and differentiation. Understanding the molecular components of these pathways and how they are regulated has thus become a major area of research in contemporary cell biology [37]. Two major pathways of intracellular signaling are based on the use of second messengers derived from the membrane phospholipid phosphatidylinositol 4,5-bisphosphate 
(PIP). PIP 1 and PIP $_{2}$ are minor components of the plasma membrane, localized to the inner layer of the phospholipid bilayer. A variety of hormones and growth factors stimulate the hydrolysis of PIP by a phospholipase $\mathrm{C}$-a reaction that produces two distinct second messengers: diacylglycerol and inositol 1,4,5-trisphosphate $\left(\mathrm{IP}_{3}\right)$, which can stimulate distinct downstream signaling pathways (protein kinase $\mathrm{C}$ and $\mathrm{Ca}^{2+}$ mobilization, respectively), so that $\mathrm{PIP}_{1}$ and $\mathrm{PIP}_{2}$ hydrolysis triggers a two-armed cascade of intracellular signaling. In the L. lancifolium trancriptome, six genes in the PIP process showed significant transcript regulation after cold stress (Figure 10). For example, two Plasma membrane intrinsic protein genes (Contig1854_All, Contig1406_All) were up-regulated by 0.58 and 1.36 -fold. Thus, the $L I P I P_{1}$ and $L I P I P_{2}$ genes in $L$. lancifolium encode plasma membrane intrinsic proteins, which contribute to triggering the protein kinase $\mathrm{C}$ and stimulating $\mathrm{Ca}^{2+}$ signaling pathways in response to low temperature [38].

Here, in L. lancifolium, protein kinase $\mathrm{C}$ including the receptor protein kinase LlCLAVATA1, a protein kinase $\mathrm{Ck} 2$ regulatory subunit and 2Serine/threonine-protein kinase cdk9 (Contig18123_All, Contig8528, Contig14527), showed up-regulated expression from 0.33 - to 1.01-fold during the cold treatment, bridging the process of signal transduction. Another two contrasting themes are apparent in the expression of the signaling pathways initiated by $\mathrm{Ca}^{2+}$ and cAMP. These two second messengers may operate towards a similar goal. The activation of phosphorylase kinase through either PKA or $\mathrm{Ca}^{2+}$ is an example of such convergence. $\mathrm{Ca}^{2+}$ and cAMP-mediated pathways may also be coordinated through $\mathrm{Ca}$-calmodulin dependent isoforms of adenylyl cyclase [36]. As an important second messenger, $\mathrm{Ca}^{2+}$ plays a vital role in the plant cold-stress response. The concentration of $\mathrm{Ca}^{2+}$ increases rapidly during cold stress, followed by a number of signals mediated by combinations of protein phosphorylation cascades [39]. As a large subfamily of plant kinases, Calcium dependent protein kinases (CDPKs) are implicated as important sensors of $\mathrm{Ca}^{2+}$ flux in plants in response to a variety of biotic and abiotic stress stimuli [40]. We identified two genes (Contig22048_All, Contig2751_All) related to CDPK, with fold changes ranging from 0.89 - to 3.28 -fold in their expression after cold stress (Figure 9); they have been demonstrated to activate a stress and ABA-inducible promoter. These results demonstrate the connection of particular CDPKs to specific signaling pathways in vivo and the usefulness of engineering CDPKs to enhance abiotic stress tolerance in L. lancifolium (Figure 10).

In many plant cells, Abscisic acid (ABA) also plays a crucial role in the cold tolerance of plants. The type $2 \mathrm{C}$ protein phosphatases ( $\mathrm{PP} 2 \mathrm{C})$, which negatively regulate ABA responses, play a key role in ABA signal transduction [8,39]. In this study, two DEGs (Contig24025_All, Contig19208_All) related to PP2C were identified that showed significant down-regulation, with fold changes ranging from 0.95 to 0.35 , in their expression after cold

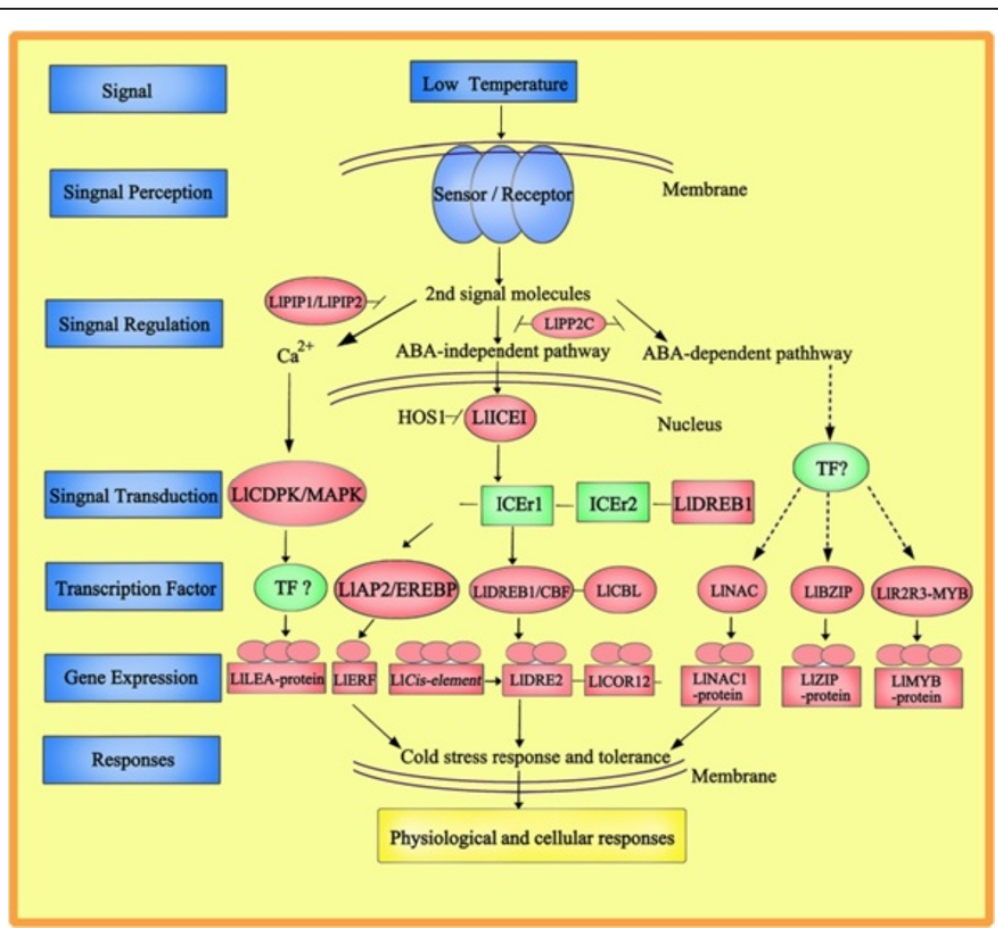

Figure 10 Models describing the signaling pathways involved in the acquisition of cold tolerance. The red box showed the indentified genes in Lilium lancifolium transcriptome; and the blue box demonstrated the unknown transcription factors and pathways in Lilium lancifolium. 
stress. The cold response has been reported to involve both ABA-dependent and independent pathways [41]. In the ABA-independent pathway, the transcription factor of DREB1 (DRE-binding protein) has also implicated in dehydration stress signaling in Arabidopsis [42]. In our research, one gene (Contig9406_All) related to LlDREB1 was identified, with a fold change of 0.31 . We presume it would trigger and induce the expression of LlCOR12 and LIDRE2 (Contig13202_All, Contig12185_All), in combination with the up-regulation of the LlCIS-element (Contig8665) by a change of 1.51 -fold. One of the big gene families that have been investigated in our study not only includes the LlAP2/EREBP (Contig10652_All) transcription factor, but also cold genes such as LIERF2, LIERF3, LIERF5, LIERF10 and LlmTERF (Contig18905_All, Contig772_All, Contig15936_All, Contig26562_All, Contig28555_All). On the other hand, the ABA-dependent pathway has genes related to transcription factors; here, LINAC and LlBZIP (Contig20596_All, Contig12014_All) in L. lancifolium were differentially expressed with fold changes of 0.46 and 1.56 , respectively, subsequently motivating the expression of the LlNAC1 and LlZIP proteins after cold exposure for $16 \mathrm{~h}$. Moreover, we also obtained a large amount of information on the MYB family from $L$. lancifolium Illumina sequencing, including the chief transcription factors $L l 1 R-M Y B 1, L l R 2 R 3-$ MYB and LIMYBR (Contig22140_All, Contig18508_All, Contig1641_All), which showed fold changes of 1.01, 6.30 , and 1.85 , respectively, and stimulated the identified cold gene LIMYB-DNAbinding protein up-regulation with a 2.92-fold change (Contig11131_All). Therefore, it is likely that the LlDREB1, LlCBL, LlAP2/EREBP, LINAC, LIBZIP and LIR2R3-MYB genes of L. lancifolium show transcription patterns under cold stress similar to those under other abiotic stresses, which are believed to activate the transcription of specific target genes in ABA signaling in guard cells (Figure 10).

Whereas the cells of prokaryotes and unicellular eukaryotes are largely autonomous, the behavior of each individual cell in multicellular plants must be carefully regulated to meet the needs of the organism as a whole. This is accomplished by a variety of signaling molecules that are secreted or expressed on the surface of one cell and bind to receptors expressed by other cells, thereby integrating and coordinating the functions of the many individual cells that make up the complex organisms [37]. In this case, cells receive and respond to signals from a cold environment, and then cold signals are received by sensors on the membrane and the second signal molecules carry them from the cytoplasm to the nucleus by the catalytic protein kinase regulatory subunit. Within the nucleus, protein kinase $\mathrm{C}$ and $\mathrm{PP} 2 \mathrm{C}$ induce the $\mathrm{Ca}^{2+}$ pathway and $\mathrm{ABA}$ signal transduction, respectively, and various transcription factors such as
LIDREB1, LIAP2/EREBP, LINAC, LIBZIP and LlR2R3$M Y B$ recruit coactivators for the transcription of inducible genes including LILEA LIERF, LIDRE2, LINAC1, LIZIP and LIMYB-DNAbinding protein, which are regulators of cellular cold tolerance and metabolic activity in short-term cold stress, and LlFAD3, Ll $\beta$-amylase genes, LIP5CS and LlCLS, which enhance adaptation processes that involve changes in the expression of transcripts related to cellular osmoprotectants, and carbohydrate metabolism during the long-term cold stress. Such regulation of gene expression plays important roles in controlling the physiological cold response, cellular morphology, proliferation, survival, and differentiation of a wide variety of plant cells, as well as being implicated in learning and memory (Figure 10).

To further study the mechanism of the cold response and acclimation of L. lancifolium, we will select specific genes and verify their functions and expression by fluorescence in situ hybridization and genetic modification technology.

\section{Conclusions}

To the best of our knowledge in L. Lancifolium, interest is further heightened by the fact that stress-regulated genes are stimulated and reacted during the short-term cold response, along with the physiological and biochemical changes during the long-term cold acclimation. The genome-wide transcriptome and physiological analysis presented in this study has expanded our knowledge of this process by identifying differentially expressed genes involved in cold regulation of carbohydrate metabolism, leaf structure and three model signaling pathways, the $\mathrm{Ca}^{2+}$ and ABA-dependent/independent pathways. Importantly, the high-resolution expression patterns presented here further our understanding of the molecular mechanisms involved in cold resistance and signal regulation for bulb flower breeding.

\section{Methods}

\section{Ethics}

This research did not involve any human subjects, human material, or human data. The field study did not involve any endangered or protected species.

\section{Plant material and total RNA isolation}

The materials used in these experiments were derived from L. lancifolium's and Oriental hybrids cultivated in the nursery of Beijing Forestry University (BJFU) $\left(116.3^{\circ} \mathrm{E}\right.$, $40.0^{\circ} \mathrm{N}$ ) under the following growth conditions: $70 \%$ relative humidity, $25^{\circ} \mathrm{C}: 18^{\circ} \mathrm{C}$, day:night temperatures, with watering every 3 days. The bulbs were collected and stored at $4^{\circ} \mathrm{C}$ for one month. Then, the bulbs were cultivated under aseptic conditions to induce leaf formation. After 4 weeks of asepsis condition, we divided the plantlets into 
two groups; the control sample (CKOh) and the coldtreated sample $(\mathrm{CT})$. Fresh leaves, stems, bulbs, and roots were subjected to a $4^{\circ} \mathrm{C}$ cold treatment for $2 \mathrm{~h}$, $8 \mathrm{~h}, 16 \mathrm{~h}, 24 \mathrm{~h}, 48 \mathrm{~h}, 4 \mathrm{~d}, 7 \mathrm{~d}, 14 \mathrm{~d}$, or $20 \mathrm{~d}$. At each time point, samples were collected and stored at $-80^{\circ} \mathrm{C}$ until RNA extraction. Total RNA was extracted from the tissues using an RNAisomate RNA Easyspin Isolation System (Aidlab Biotech, Beijing, China) according to the manufacturer's instructions. The quality of RNA was verified using a 2100 Bioanalyzer (Agilent Technologies, Santa Clara, CA, USA). The RNA concentration was at least $160 \mu \mathrm{g} / \mathrm{mm}^{3}$ in all samples. To prepare cDNA, we used a pooled RNA mixture containing $60 \mu \mathrm{g}$ RNA from each sample.

\section{cDNA library preparation and transcriptome sequencing}

Illumina sequencing was conducted using the Solexa mRNA-Seq platform at the Shanghai manufacturer's instructions (Illumina, San Diego, CA, USA). Briefly, we used magnetic beads with oligo(dT) to isolate poly(A) mRNA after isolating total RNA from $L$. lancifolium leaves in the control $(0 \mathrm{~h})$ and after $2 \mathrm{~h}$ and $16 \mathrm{~h}$ of cold treatment. Second-strand cDNA was synthesized using appropriate buffers, dNTPs, RNase $\mathrm{H}$, and DNA polymerase I. ShoBiotechnology Corporation (SBC), Shanghai, China (http://www.ebioservice.com) according to the rt fragments were depurated with a QiaQuick PCR extraction kit (Qiagen, Hilden, Germany) and resolved with an elution buffer for end repair and by addition of poly(A). For PCR amplification, we selected suitable fragments as templates based on the results of agarose gel electrophoresis. The library was sequenced using an Illumina $\mathrm{HiSeq}^{\mathrm{mi}} 2000$. Because raw reads produced from sequencing machines contain low-quality reads that negatively affect subsequent bioinformatics analyses, we discarded reads with adapters, those with more than 5\% unknown nucleotides, and those of low quality ( $\leq 20 \%$ of the bases with a quality score $(\mathrm{Q}) \leq 10)$ using an in-house Perl script. The average proportion of clean reads in each sample was $89.6 \%-91.7 \%$. The clean reads were used for further analyses.

\section{Analysis of Illumina transcriptome sequencing results}

De novo assembly was carried out using scaffolding contig methods with CLC Genomics Workbench (version: 5.5) with the default parameters, and a minimum contig length of $\geq 400$. The assembled de novo sequences were designated as primary unigenes. After assessing the different K-mer sizes, we found that 29-mer yielded the best assemblies and so this size class was selected to construct the de Bruijn graph. Primary unigenes from UniGene of three samples' were assembled using CAP3 $E S$, yielding final unigenes. Assembled final unigenes were used for BLASTx searches (E-value $<1 \mathrm{e}-5$ ) against the UniProt database (date: 2013.04) and the Swiss-Prot protein database (date: 2013.05) (http://www.expasy.ch/ sprot), which has the largest and most particular protein annotation database (approximately including 24,889,084). To functionally annotate sequences, we used Blast2GO program (Conesaet et al., 2005) to assign gene ontology (GO) terms (http://www.geneontology.org). Also, to predict and classify possible functions, 13705 unigene sequences were aligned to 25 Clusters of Orthologous Groups (COGs) in the COG database (http://www.ncbi. nlm.nih.gov/COG). Kyoto Encyclopedia of Genes and Genomes Pathway (KEGG; http://www.genome.jp/kegg) annotations were carried out according to the KEGG database using BLASTx (E-value threshold $10^{-5}$ ).

\section{Bioinformatics for functional annotation of differential gene expression}

A rigorous algorithm to identify differentially expressed genes was developed based on the method of Audic et al. (1997). The false discovery rate (FDR) was used to determine the threshold of the P-value in multiple tests and analyses. We used an FDR of $<0.001$ and the absolute value of $\log _{2}$ (ratio) $\geq 2$ as thresholds to define significantly different gene expression [43]. For further analyses, we used an additional criterion, which involved using only differentially expressed genes (DEGs) with a minimum of a four-fold change in expression.

\section{Transcription factors analysis}

Transcription factors were predicted according to protein sequences obtained from CDS predictions. We used HMM search to search for plant transcription factor domains (http://plntfdb.bio.uni-potsdam.de/v3.0/) and classified unigenes according to gene family information.

\section{Real-time quantitative PCR verification}

Total RNA was isolated from the leaf, stem, bulbs, and roots of lily plants subjected to $4^{\circ} \mathrm{C}$ cold treatments, as described above. First-strand cDNA synthesis was performed using Superscript II reverse transcriptase (Invitrogen, Carlsbad, CA, USA) according to the manufacturer's instructions, using $1 \mu \mathrm{g}$ total RNA and oligo(dT) primers. qRT-PCR was performed using a Rotor-Gene 3000 realtime PCR detection system (Qiagen) using $\mathrm{SYBR}^{\circ} \mathrm{qPCR}$ Mix (Toyobo, Tokyo, Japan) according to the manufacturer's protocol. The primers used in this study were designed with Beacon Designer (Premier, Palo Alto, CA, USA) and are listed in Table 1. Real-time PCRs was carried out using prepared cDNA $(80 \mu \mathrm{g})$ with each set of primers and probe and $\mathrm{iQ}^{\mathrm{Tw}} \mathrm{SYBR}^{\circ}$ Green Supermix (Cat. No.170-8882, Bio-Rad, Hercules, CA, USA). The PCR cycling conditions were as follows: $95^{\circ} \mathrm{C}(30 \mathrm{~s}), 60^{\circ} \mathrm{C}(30 \mathrm{~s})$, and $72^{\circ} \mathrm{C}(15 \mathrm{~s})$. All reactions were performed in biological triplicates. Relative mRNA levels were calculated using the $2^{-\triangle \Delta \mathrm{Ct}}$ method [44] against the internal reference gene 
TIP1, with expression in CT $0 \mathrm{~h}$ used as the internal control. The sequences of primers used for QRT-PCR are listed in Table 4.

\section{Heat-map generation}

A heat-map of legume-specific genes and the genes with the highest transcript levels was generated using the heat-map function in the gplots CRAN library. After excluding legume-specific genes that did not have a RPKM normalized $\log _{2}$-transformed transcription count greater than zero in at least one tissue, 315 genes remained. The LSGs were taken from the Glyma1.01 gene set. The genes with the highest transcript levels were determined based on the sum of raw counts in all tissues. Boxes were added to reveal clusters of genes with similarly expression in specific tissues. (Additional file 1: Table S1) showed additional details indicating the gene represented by each cell in the heat-map.

\section{Leaf structural characteristics}

To investigate the internal anatomy of leaves, sections were cut through the leaf midrib. The proximal halves of individual leaves were fixed in $0.3 \mathrm{mg} / \mathrm{cm}^{3}$ paraformaldehyde, 5\% ethanoic acid, and 50\% ethanol, and then dehydrated in a graded ethanol (50\%-95\%) series. Sections ( $1 \mu \mathrm{m}$ thick) were cut with a micrometre (Ultracut UCT, Leica Microsystems, Welzlar, Germany), stained with toluidine, and imaged with a microscope and imaging system (Optiphot 2 with DS-L1, Nikon, Tokyo, Japan). The cut surface was mid-way between the midrib and margin, near the widest point of the leaf.

\section{Carbohydrate and electrical conductivity analysis}

Total soluble proteins, soluble sugars, starch, and malondialdehyde (MDA) content were determined using leaf tissue from plants subjected to 1 to 20 days of cold treatment. The leaf tissue was collected and stored at $-80^{\circ} \mathrm{C}$. Carbohydrate content and electrical conductivity were measured as described by Gilmour [45]. Soluble sugars were analyzed using the phenol-sulfuric acid method. Soluble proteins were determined using the Coomassie brilliant blue colorimetric method. Starch was quantified using the anthronesulfuric acid method and MDA content was determined using the thiobarbituric acid method. Absorbance was measured using a plate reader (POLARstar OPTIMA, BMG Labtech, Offenburg, Germany). Electrical conductivity was measured by the bath method using a desktop meter (EC3175-307, JENCO, San Diego, CA, USA).

\section{Availability of supporting data}

The data sets supporting the results of this article are available in the [NCBI GenBank] repository, [unique persistent identifier (KJ467617, KJ467618, KJ467619, KJ467620, KJ467621, KJ467622, KJ467623, KJ467624,
KJ489024, KJ489025, KJ489026,) and hyperlink to datasets in http://www.ncbi.nlm.nih.gov/genbank/].

And also, the other data sets supporting the results of this article are included within the article and its additional files TXTS3.

\section{Additional files}

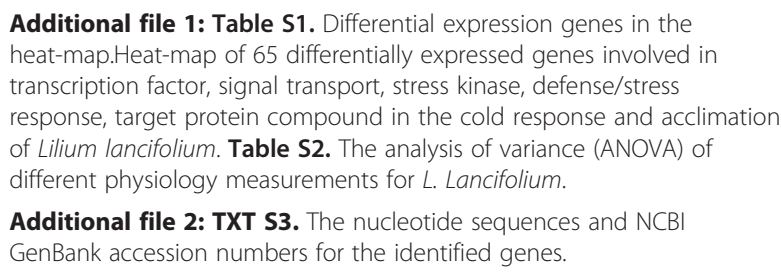

Additional file 2: TXT S3. The nucleotide sequences and NCBI GenBank accession numbers for the identified genes.

\section{Abbreviations}

BLAST: Basic local alignment search tool's Control 0 h; CT: Cold treatment: COG: Cluster of orthologous group; DEGs: Differentially expressed geneses Expressed sequence tag; GO: Gene ontology; KEGG: Kyoto encyclopedia of genes and genomes; MDA: Malondialdehyde; qRT-PCR: Real-time quantitative reverse transcription PCR; SP: Swiss-Prot protein database; SOM: Self-organizing map; TF: Transcription factor.

\section{Competing interests}

The authors declare that they have no competing interests.

\section{Author's contributions}

JMW carried out the molecular genetic studies, participated in the sequence alignment and drafted the manuscript. YY carried out the immunoassays. $\mathrm{XHL}$ participated in the sequence alignment. JMW, JH participated in the design of the study and performed the statistical analysis. JHG, QW conceived of the study, and participated in its design and coordination and helped to draft the manuscript. All authors read and approved the final manuscript.

\section{Acknowledgements}

This work was supported by the '863' research program (Grant No. 2011AA10020804), China National Natural Science Foundation (Grant No. 31071815 and No. 31272204), and the D. Programs Foundation of the Ministry of Education of China (Grant No. 20110014110006). We thank Professor Guijun Yan for his insightful comments on the paper. We also thank Heyan Cao, Shunchao Xu, Jie Guan, Wenqi Li and Chengke Li, who studied in our laboratory.

\section{Author details}

${ }^{1}$ College of Landscape Architecture \& China National Engineering Research Center for Floriculture, Beijing Forestry University, No.35 Qinghua East Road Haidian District, Beijing 100083, China. ${ }^{2}$ College of soil and water conservation, Beijing Forestry University, No.35 Qinghua East Road Haidian District, Beijing 100083, China.

Received: 18 October 2013 Accepted: 7 March 2014 Published: 17 March 2014

\section{References}

1. Bray EA, Bailey-Serres J, Weretilnyk E: Responses to abiotic stresses. Chapter 22. In Responses to Abiotic Stresses. Edited by Gruissem W, Buchannan B, Jones R. Rockville, MD: American Society of Plant Physiologists; 2000:1158-1249.

2. Kreps JA, Wu Y, Chang H-S, Zhu T, Wang X, Harper JF: Transcriptome changes for Arabidopsis in response to salt, osmotic, and cold stress. Plant Physiol 2002, 130:2129-2141.

3. Cheng C, Yun K-Y, Ressom HW, Mohanty B, Bajic VB, Jia Y, Yun SJ, Reyes $B G D$ : An early response regulatory cluster induced by low temperature and hydrogen peroxide in seedlings of chilling- tolerant japonica rice. BMC Genomics 2007, 8(7):175 
4. Christov NK, Yoneyama S, Shimamoto Y, Imai R: Differential expression of wheat genes during cold acclimation. Cytol Genet 2007, 41(1):142-150.

5. Cao PX, Song J, Zhou CJ, Weng ML, Liu J, Wang FX, Zhao F, Feng DQ, Wang $B$ : Characterization of multiple cold induced genes from Ammopiptanthus mongolicus and functional analyses of gene AmEBP1. Plant Mol Biol 2009, 69(6):529-539.

6. Allen GJ, Chu SP, Schumacher K, Shimazaki CT, Vafeados D, Kemper A, Hawke SD, Tallman G, Tsien RY, Harper JF: Alteration of stimulus-specific guard cell calcium oscillations and stomatal closing in Arabidopsis det3 mutant. Science 2000, 289:2338-2342.

7. Seki M, Narusaka M, Abe H, Kasuga M, Yamaguchi-Shinozaki K, Carninci P, Hayashizaki Y, Shinozaki K: Monitoring the expression pattern of 1300 Arabidopsis genes under drought and cold stresses by using a full-length cDNA microarray. Plant Cell 2011, 13(6):61-72

8. Thomashow MF: Freezing tolerance genes and regulatory mechanisms. Ann Rev Plant Physiol. Plant Mol Biol 1999, 50:571-599.

9. Fowler S, Thomashow MF: Arabidopsis transcriptome profiling indicates that multiple regulatory pathways are activated during cold acclimation in addition to the CBF cold response pathway. Plant Cell 2002, 14:1675-1690.

10. Zhang G, Guo G, Hu X, Zhang Y, Li Q, Li R, Zhuang R, Lu Z, He Z, Fang X Chen $L$ : Deep RNA sequencing at single base-pair resolution reveals high complexity of the rice transcriptome. Genome Res 2010, 20(2):646-654.

11. Raherison E, Rigault P, Caron S, Poulin P, Boyle B, Verta JP, Giguere I, Bomal C, Bohlmann J, MacKay J: Transcriptome profiling in conifers and the PiceaGenExpress database show patterns of diversification within gene families and interspecific conservation in vascular gene expression. BMC Genomics 2012, 13:434-449.

12. Qiu Q, Ma T, Hu Q, Liu B, Wu Y, Zhou H, Wang Q, Wang J, Liu J: Genomescale transcriptome analysis of the desert poplar, Populus euphratica. Tree Physiol 2011, 31(4):452-461.

13. Xu DL, Long H, Liang JJ, Zhang J, Chen X, Li JL, Pan ZF, Deng GB, Yu MQ: De novo assembly and characterization of the root transcriptome of Aegilops variabilis during an interaction with the cereal cyst nematode. BMC Genomics 2012, 13:133-137.

14. Cong HQ, Li ZY, Xu L: Characterizing developmental and inducible differentiation between juvenile and adult plants of Aechmea fasciata treated with ethylene by transcriptomic analysis. Plant Growth Regul 2013, 69:247-257.

15. Trick M, Long Y, Meng J, Bancroft I: Single nucleotide polymorphism (SNP) discovery in the polyploid Brassica napus using Solexa transcriptome sequencing. Plant Biotechnol J 2009, 7(4):334-346.

16. Li P, Ponnala L, Gandotra N, Wang L, Si Y, Tausta SL, Kebrom TH, Provart N, Patel R, Myers CR, Reidel EJ, Turgeon R, Liu P, Sun Q, Nelson T, Brutnell TP: The developmental dynamics of the maize leaf transcriptome. Nat Genet 2010, 42(12):1060-1067.

17. Zhang J, Liang S, Duan J, Wang J, Chen S, Cheng Z, Zhang Q, Liang X, Li Y. De novo assembly and Characterisation of the Transcriptome during seed development, and generation of genic-SSR markers in Peanut (Arachis hypogaea L.). BMC Genomics 2012, 13:90.

18. Gahlan P, Singh HR, Shankar R, Sharma N, Kumari A, Chawla V, Ahuja PS, Kumar S: De novo sequencing and characterization of Picrorhiza kurrooa transcriptome at two temperatures showed major transcriptome adjustments. BMC Genomics 2012, 13:126.

19. Pilar M, Gregogy T, Mccollum MP: Transcriptome profiling of grapefruit flavedo following exposure to low temperature and conditioning treatments uncovers principal molecular components involved in chilling tolerance and susceptibility. Plant Cell Environ 2008, 31:752-768.

20. Pang $T, Y e C Y, X i a ~ X L$, Yin WL: De novo sequencing and transcriptome analysis of the desert shrub, Ammopiptanthus mongolicus, during cold acclimation using Illimina/Solexa. BMC Genomic 2013, 14:488.

21. Chen JH, Song YP: Genome-Wide analysis of gene expression in response to drought stress in Populus simomnii. Plant Mol Biol Rep 2013, 31:946-996.

22. Kawamura $Y$, Uemura M: Mass spectrometric approach for identifying putative plasma membrane proteins of Arabidopsis leaves associated with cold acclimation. Plant J 2003, 36:141-154.

23. Moellering ER, Muthan B, Benning C: Freezing tolerance in plants requires lipid remodeling at the outer chloroplast membrane. Science 2010 , 330:226-228.
24. Degenkolbe T, Giavalisco P, Zuther E, Seiwert B, Hincha DK, Willmitzer L: Differential remodeling of the lipidome during cold acclimation in natural accessions of Arabidopsis thaliana. Plant J 2012, 32:972-982.

25. Gibson S, Arondel V, Iba K, Somerville C: Cloning of a temperatureregulated gene encoding a chloroplast omega-3 desaturase from Arabidopsis thaliana. Plant Physiol 1994, 106:1615-1621.

26. Gorsuch PA, Pandey S, Atkin OK: Temporal heterogeneity of cold acclimation phenotypes in Arabidopsis leaves. Plant Cell Environ 2010, 33:44-258.

27. Ashraf M, Foolad MR: Roles of glycine betaine and proline in improving plant abiotic stress resistance. Environ Exp Bot 2007, 59:206-216.

28. Verbruggen $N$, Hermans C: Proline accumulation in plants: a review. Amino Acids 2008, 35:753-759.

29. Tunnacliffe A, Wise MJ: The continuing conundrum of the LEA proteins. Naturwissenschaften 2007, 94:791-812

30. Rajesh S, Manickam A: Prediction of functions for two LEA proteins from mung bean. Bioinformation 2007, 1:133-138.

31. Qiu ZB, Wan LC: The regulation of cambial activity in Chinese fir (Cunninghamia lanceolata) involves extensive transcriptome remodeling. New Phytologist 2013, 199:627-870.

32. Xin Z: Cold comfort farm: the acclimation of plants to freezing temperatures. Plant Cell Environ 2001, 23:893-902.

33. Porankiewicz J, Wang J, Clarke AK: New insights into the ATP-dependent Clp protease: Escherichia coli and beyond. Mol Microbio/ 1999, 32:449-458.

34. Wang B, Guo G, Wang C, Lin Y, Wang X, Zhao M, Guo Y, He M, Zhang Y, Pan L: Survey of the transcriptome of Aspergillus oryzae via massively parallel mRNA sequencing. Nucleic Acids Res 2010, 38:5075-5087.

35. Srichuwonga S, Isonoa N, Mishimab T, Hisamatsua M: Structure of lintnerized starch is related to X-ray diffraction pattern and susceptibility to acid and enzyme hydrolysis of starch granules. Int J Biol Macromol 2007, 37:115-121

36. Gomperts B, Kramer IM, Tatham PER: Signal transcription. Beijing Sci Publ 2007, 7:145-156. ISBN 978-7-03-018217-3.

37. Cooper GM, Hausman RE: The cell: a molecular approach. Beijing Sci Publ 2012, 15:603-605. ISBN 978-7-03-031324-9.

38. Donald WH, Rebecca B: Regulation of Cardiac $\mathrm{Na}^{+}, \mathrm{Ca}^{2+}$ Exchange and

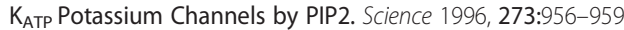

39. Saijo Y, Hata S, Kyozuka J, Shimamoto K, Izui K: Over-expression of a single $\mathrm{Ca} 2+-$ dependent protein kinase confers both cold and salt/drought tolerance on rice plants. Plant J 2000, 23:319-327.

40. Ludwig AA, Romeis T, Jones JDG: CDPK-mediated signalling pathways: specificity and cross-talk. J Exp Bot 2004, 55:181-188.

41. Agarwal PK, Jha B: Transcription factors in plants and ABA dependent and independent abiotic stress signalling. Biol Plantarum 2010, 54:201-212.

42. Yoshida R, Hobo T, Ichimura K, Mizoguchi T, Takahashi F, Aronso J, Ecker JR, Shinozaki K: ABA-activated SnRK2 protein kinase is required for dehydration stress signaling in Arabidopsis. Plant Cell Physiol 2002, 43:1473-1483.

43. Benjamini $Y$, Yekutieli D: The control of the false discovery rate in multiple testing under dependency. Ann Stat 2001, 29:1165-1188.

44. Gilmour SJ, Zarka DG, Stockinger EJ, Salazar MP, Houghton JM, Thomashow MF: Low temperature regulation of the Arabidopsis CBF family of AP2 transcriptional activators as an early step in cold-induced COR gene expression. Plant J 1998, 16:433-442.

doi:10.1186/1471-2164-15-203

Cite this article as: Wang et al:: Transcriptome profiling of the cold response and signaling pathways in Lilium lancifolium. BMC Genomics 2014 15:203. 